\title{
Synthesis and biological evaluation of benzopyran analogues bearing class III antiarrhythmic pharmacophores
}

\author{
Maria Koufaki, ${ }^{\mathrm{a}, *}$ Christina Kiziridi, ${ }^{\mathrm{a}}$ Panagiota Papazafiri, ${ }^{\mathrm{b}}$ Athanasios Vassilopoulos, ${ }^{\mathrm{b}}$ \\ Andràs Varró, ${ }^{\mathrm{c}}$ Zsolt Nagy, ${ }^{\mathrm{c}}$ Attila Farkas ${ }^{\mathrm{c}}$ and Alexandros Makriyannis ${ }^{\mathrm{d}}$ \\ ${ }^{a}$ Institute of Organic and Pharmaceutical Chemistry, National Hellenic Research Foundation, \\ 48 Vas. Constantinou Avenue, 11635 Athens, Greece \\ ${ }^{\mathrm{b}}$ Department of Animal and Human Physiology, School of Biology, University of Athens, Panepistimiopolis, 15784 Athens, Greece \\ ${ }^{\mathrm{c}}$ Department of Pharmacology and Pharmacotherapy University of Szeged, Szeged Dom ter 12 H-6720, Hungary \\ ${ }^{\mathrm{d}}$ Northeastern University, Center for Drug Discovery, 360 Huntington Avenue, 116 Mugar Hall, Boston, MA 02115, USA
}

Received 13 March 2006; revised 25 May 2006; accepted 31 May 2006

Available online 19 June 2006

\begin{abstract}
We have synthesized a series of compounds combining the hydroxy-benzopyran ring of vitamin E with the methylsulfonylaminophenyl group of class III antiarrhythmic drugs, connected through tertiary amine moieties. Evaluation of the antiarrhythmic and antioxidant activity of the new compounds was carried out on isolated rat heart preparations using the non-recirculating Langendorff mode. The new analogues were present, at $10 \mu \mathrm{M}$ concentration, during ischemia and reperfusion. Selected compounds were further studied by a conventional microelectrode method in order to get insight into their cellular mode of action. The most active compound, $\quad N$-[4-[2-[[2-(3,4-dihydro-6-hydroxy-2,2,7,8-tetramethyl-2 $\mathrm{H}$-1-benzopyran-5-yl)ethyl] methylamine]ethyl]phenyl]methanesulfonamide (19a), reduces premature beats, prolongs QT and QRS intervals during ischemia and reperfusion, and reduces MDA content, leading to a fast recovery of the heart. In addition, it exhibits moderate class III antiarrhythmic action. (C) 2006 Elsevier Ltd. All rights reserved.
\end{abstract}

\section{Introduction}

Arrhythmias account for nearly one quarter of all cardiovascular-related deaths. The majority of such deaths is caused by the degeneration of a normal cardiac rhythm into ventricular tachycardia (VT) followed by ventricular fibrillation (VF). ${ }^{1}$ The shape of the action potential of the heart cells is strongly controlled by the correct interplay of ion channels. ${ }^{2}$ The main ion channels contributing to the action potential are sodium, potassium, and calcium channels. Changes in the ionic mechanism responsible for the generation and the propagation of the normal action potential can cause abnormalities in the electrical activity of the heart.

Although cardiac rhythm disturbances may be the result of a variety of pathophysiological conditions, coronary

Keywords: Benzopyran analogue; Antiarrhythmic agents; Ischemia/ reperfusion; Cellular electrophysiology.

* Corresponding author. Tel.: +30 210 7273818; fax: +30 210

7273831; e-mail: mkoufa@eie.gr artery disease which has resulted in prior ischemia is most important. Myocardial ischemia causes profound alterations in normal cardiac electrophysiology and cellular metabolism, precipitating ventricular arrhythmias or fibrillation. ${ }^{3}$ The establishment of blood flow to the myocardium, by procedures such as thrombolysis, angioplasty and coronary bypass surgery, reduces the mortality of ischemic tissues. However, the reactive oxygen species (ROS), produced upon the readmission of oxygenated blood into the ischemic myocardium (reperfusion), ${ }^{4}$ affect selective permeability of cell membranes, leading to the development of life-threatening ventricular arrhythmias and/or fibrillation.

Moreover, post-operative atrial fibrillation is a common complication after open heart surgery; it increases morbidity, hospital stay, and costs. ${ }^{5}$ Pharmacologic strategies and regimens aimed at preventing post-operative atrial fibrillation are necessary to patients undergoing open heart operations. The prevalence of arrhythmia in the population is increasing as more people survive for longer with cardiovascular disease. ${ }^{6}$ Since many patients experience a decrease in physical performance as 
well as a diminished quality of life, there is still a need for antiarrhythmic drug therapy.

According to the classification of Vaughan Williams (1970), based on electrophysiological actions, the antiarrhythmic drugs can be defined by four classes: ${ }^{7}$ class I consist of antiarrhythmic agents that block sodium channels, reducing the maximum increase rate of depolarization $\left(V_{\max }\right)$. Class II are $\beta$-blockers. Class III act through delaying repolarization of cardiac myocytes and thus cause a lengthening of APD (potassium-channel blockers). Class IV block calcium currents in cardiac tissue.

In the case of antiarrhythmic drugs the delicate balance between drug efficacy and unexpected adverse side effects is narrower than in any other class of therapeutic agents. Concerning class I antiarrhythmics, controlled trials suggested the effectiveness of routine lidocaine (class Ib) prophylaxis in preventing ventricular fibrillation due to acute myocardial infarction. ${ }^{8,9}$ However, in 1980 CAST (Cardiac Arrhythmia Suppression Trial), with the drugs encainide and flecainide (class Ic), uncovered the inefficacy and even proarrhythmic risk of sodium channel blockers in post-infarction patients. To circumvent the problems with class I antiarrhythmics, pharmacological and clinical research shifted toward the class III agents. ${ }^{10}$ Amiodarone (combining class IIV properties), ${ }^{11 \mathrm{a}, 11 \mathrm{~b}} \mathrm{~d}, 1$-sotalol (class II, III) ${ }^{1 \mathrm{cc}}$ and ambasilide (class II, III), azimilide (class I, III, IV) are complex class III compounds, while 'pure' class III agents are d-sotalol, dofetilide, ibutilide.

Sotalol, amiodarone, ibutilide, and dofetilide are moderately effective in patients with chronic atrial fibrillation. However, amiodarone appears to be most efficacious. Moreover, amiodarone and dofetilide are safe in patients who have had a myocardial infarction and those with heart failure. The safety of commercially available $\mathrm{d}, 1$-sotalol in these patients is poorly understood. Torsades de pointes is the most serious adverse effect of sotalol and dofetilide. Amiodarone has minimal proarrhythmic risk but has numerous noncardiac toxicities that require frequent monitoring. ${ }^{12 a, 12 b}$ Dronedarone a noniodinated benzofuran derivative has been shown to be more effective in vivo than amiodarone in several arrhythmia models, particularly in preventing ischemiaand reperfusion-induced ventricular fibrillation and in reducing mortality. However, further experimental studies and long-term clinical trials are required to provide additional evidence of efficacy and safety of this drug. ${ }^{12 \mathrm{c}}$ Azimilide statistically reduced the incidence of new atrial fibrillation in recent survivors of myocardial infarction at high risk for sudden cardiac death. ${ }^{13}$ In addition, class III antiarrhythmic agents are increasingly being used as adjunct therapy to decrease the frequency of ICD discharges in patients with ventricular arrhythmias and implantable cardioverter defibrillators (ICDs). ${ }^{14}$ The antiarrhythmic efficacy of most pure class III drugs is compromised by their inherent property to induce excessive lengthening of the action potential and their inability to prolong the action potential when most needed, namely during tachycardia. Overall, an ideal antiarrhythmic agent does not exist, and drug selection should be highly individualized. ${ }^{15,16}$

Thus, it is important to develop therapeutic agents which could improve heart function with minimal side effects. We have previously synthesized ${ }^{17}$ a series of hybrid compounds combining the pharmacophoric redox moiety of vitamin $\mathrm{E}$ and key features responsible for the antiarrhythmic properties of the class I antiarrhythmics procainamide and lidocaine. Some of these compounds, at concentrations of $30-100 \mu \mathrm{M}$, prolonged QRS intervals during reperfusion and enhanced the post-ischemic recovery without inducing ventricular fibrillations. Moreover, there was no evidence in our experiments for drug-induced proarrhythmia.

Based on this experience in antiarrhythmic field, we were interested in continuing our work and to focus our activities on the synthesis of novel cardioprotective compounds with improved efficacy in the treatment of life-threatening arrhythmias. Thus, we synthesized series of molecules that combine the hydroxy-benzopyran ring of vitamin $\mathrm{E}$ with the methylsulfonylaminophenyl moiety of class III antiarrhythmic drugs.

Specifically, the new compounds combine pharmacophores identified for the most active class III antiarrhythmics. Thus, they contain two aromatic rings, one methylsulfonyl amino group and at least one tertiary amine, such as a 1,4-piperazine or methylamine moiety.

Evaluation of the antiarrhythmic and antioxidant activity of the new compounds was carried out on isolated rat heart preparations using the non-recirculating Langendorff mode. The new analogues were present, at $10 \mu \mathrm{M}$ concentration, during ischemia and reperfusion. Selected compounds were further studied by a conventional microelectrode method in order to get insight into their cellular mode of action.

\section{Chemistry}

The synthesis of the disubstituted piperazine derivatives $\mathbf{5 a}-\mathbf{e}$ is depicted in Scheme 1. Analogues 2a-e were synthesized by alkylation of the appropriate monosubstituted piperazines (prepared by the appropriate bromides or acid chloride and 8-fold excess of piperazine) with bromide $\mathbf{1}^{18}$ in the presence of $\mathrm{K}_{2} \mathrm{CO}_{3}$ and TBAI in $\mathrm{CH}_{3} \mathrm{CN}$ (compounds 2a-c,e) or DMF (compound 2d). Reduction of the nitro group using $\mathrm{NaBH}_{4}$ and $\mathrm{CuCl}$ gave analogues $\mathbf{3 a}-\mathbf{e}$, which in turn were converted to the corresponding methanesulfonamides $4 \mathrm{a}-\mathbf{e}$ using $\mathrm{CH}_{3} \mathrm{SO}_{2} \mathrm{Cl}$ in pyridine. Deprotection of the chroman hydroxyl group was achieved using $\left.\mathrm{BF}_{3} \cdot \mathrm{S}_{(} \mathrm{CH}_{3}\right)_{2}$ in $\mathrm{CH}_{2} \mathrm{Cl}_{2}$, to afford the methanesulfonamides $\mathbf{5 a}-\mathbf{e}$.

The 1-[(benzopyran-5-yl)ethyl]piperazine analogue $\mathbf{1 2}$ is synthesized as shown in Scheme 2. Wittig reaction of aldehyde 6 with $\mathrm{Ph}_{3} \mathrm{P}^{+} \mathrm{CH}_{2} \mathrm{OCH}_{3} \mathrm{Cl}^{-}$in the presence of $t$-BuOK gave the enol ether 7 which upon hydroly$\operatorname{sis}^{19}$ afforded aldehyde $\mathbf{8}$. Reductive amination of $\mathbf{8}$ with 1-(4-nitrophenyl)-piperazine produced the nitro 

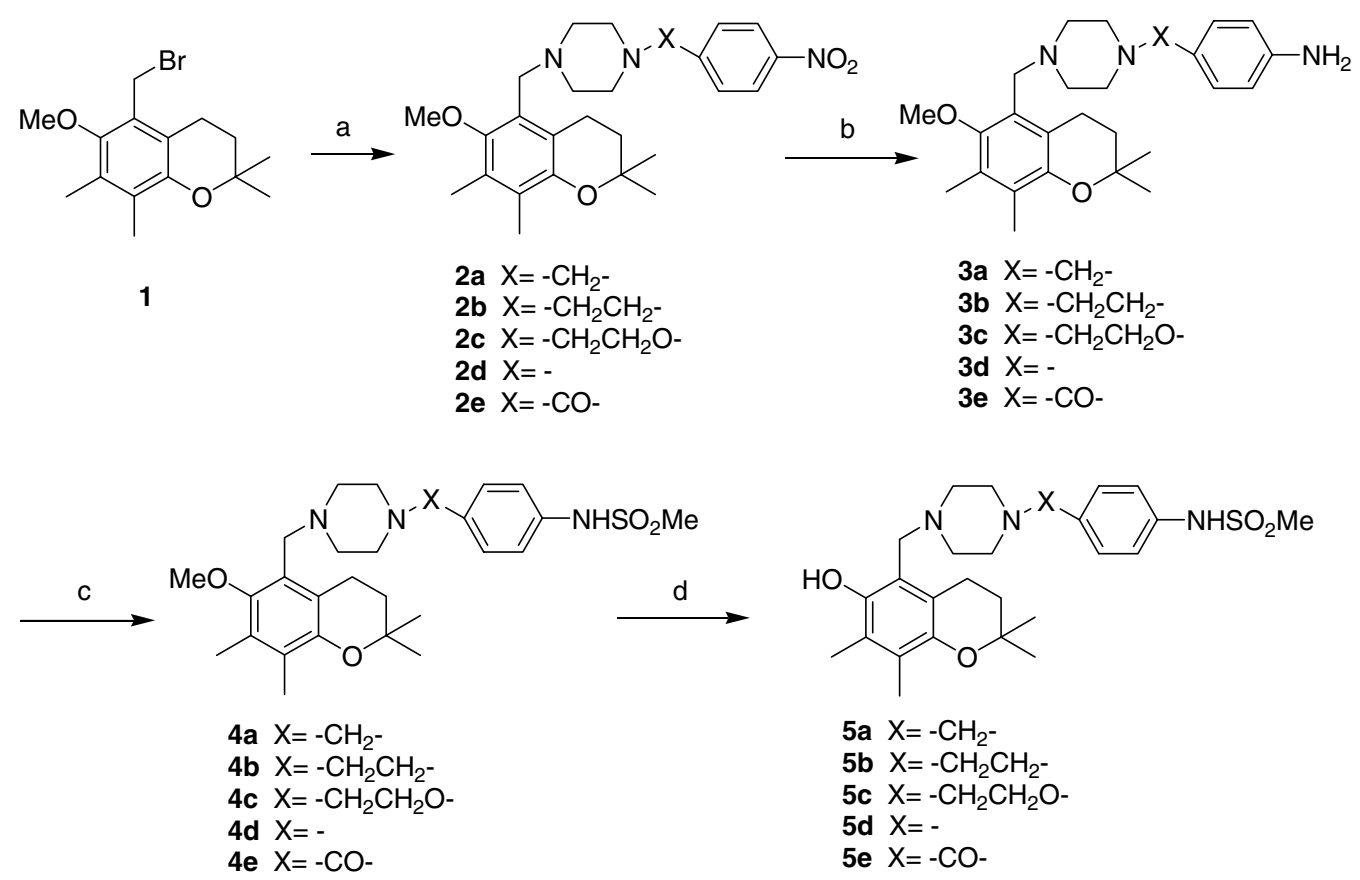

Scheme 1. Reagents and conditions: (a) 4-substituted piperazine, $\mathrm{K}_{2} \mathrm{CO}_{3}$, TBAI, anhyd $\mathrm{CH}_{3} \mathrm{CN}$; (b) 1-(4-nitrophenyl)piperazine, $\mathrm{K}_{2} \mathrm{CO}$, $\mathrm{TBAI}$,

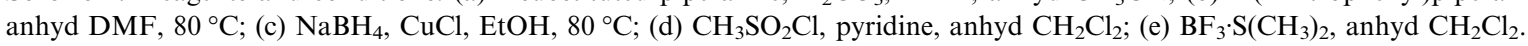<smiles>[3H]c1c(C)c(OC)c(/C=C\OC)c2c1OC(C)(C)CC2CCCC</smiles>

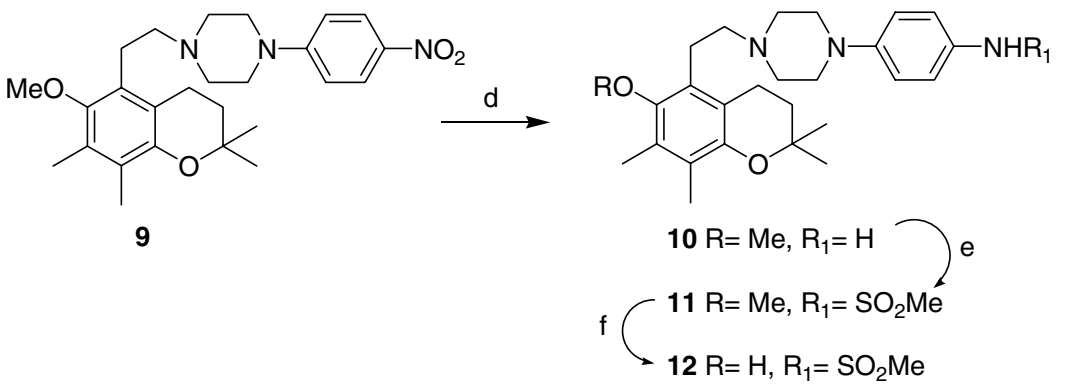

Scheme 2. Reagents and conditions: (a) $\mathrm{Ph}_{3} \mathrm{P}^{+} \mathrm{CH}_{2} \mathrm{OCH}_{3} \mathrm{Cl}^{-}, t$-BuOK, anhyd THF; (b) p-toluenesulfonic acid, dioxane, $\mathrm{H}_{2} \mathrm{O}$; (c) 1-(4-

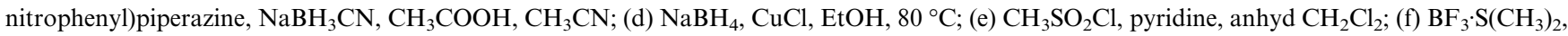
anhyd $\mathrm{CH}_{2} \mathrm{Cl}_{2}$.

analogue 9 which was converted to methanesulfonamide 12 using the synthetic procedure described above for compounds 5a-e.

The synthesis of methylamino derivatives $19 \mathbf{a}, \mathbf{b}$ is depicted in Scheme 3. Condensation of aldehyde $\mathbf{6}$ with $\mathrm{CH}_{3} \mathrm{NO}_{2}$, in the presence of $\mathrm{CH}_{3} \mathrm{COONH}_{4}$, followed by reduction using $\mathrm{LiAlH}_{4},{ }^{20}$ gave amine 14, which in turn was alkylated with 4-nitrophenyl or 2-(4-nitro-phenoxy)ethyl bromide to afford amines 15a,b. Methylation of the secondary amines using $\mathrm{HCHO} 36 \%$ and $\mathrm{HCOOH}^{21}$ afforded nitro compounds $\mathbf{1 6 a}, \mathbf{b}$ which were converted to methanesulfonamides $19 \mathbf{a}, \mathbf{b}$.
For the synthesis of the constrained derivative $\mathbf{2 2}$ (Scheme 4), bromide 1 and 6-nitroindoline were used as starting materials. Analogue $\mathbf{2 1}$ was synthesized by reductive alkylation of 6-nitroindoline with aldehyde $\mathbf{8}$ and then converted to the final compound $\mathbf{2 3}$.

\section{Results and discussion}

The antiarrhythmic activity of the new compounds, at a concentration of $10 \mu \mathrm{M}$, is shown in Table 1 and it is expressed as incidence of premature beats during the first $10 \mathrm{~min}$ of reperfusion. The antioxidant activity is 
<smiles>[Y4]c1c(C)c(OC)c2c(c1/C=C\[N+](=O)[O-])CCC(C)(CC)O2</smiles>

13

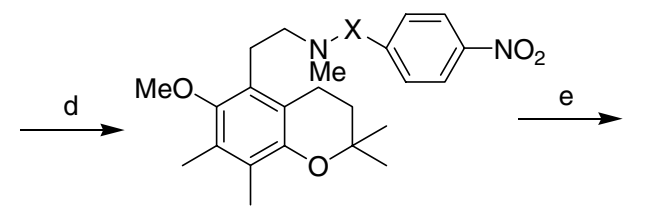

16a $\mathrm{X}=-\mathrm{CH}_{2} \mathrm{CH}_{2}-$

$16 \mathbf{b} X=-\mathrm{CH}_{2} \mathrm{CH}_{2} \mathrm{O}-$

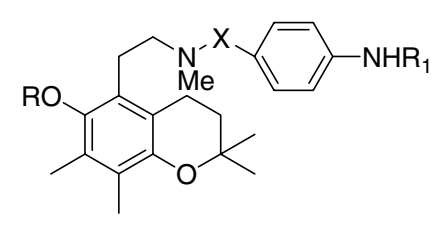

17a $\mathrm{R}=\mathrm{Me}, \mathrm{R}_{1}=\mathrm{H}, \mathrm{X}=-\mathrm{CH}_{2} \mathrm{CH}_{2}-$

17b $\mathrm{R}=\mathrm{Me}, \mathrm{R}_{1}=\mathrm{H}, \mathrm{X}=-\mathrm{CH}_{2} \mathrm{CH}_{2} \mathrm{O}-$

18a $\mathrm{R}=\mathrm{Me}, \mathrm{R}_{1}=\mathrm{SO}_{2} \mathrm{Me}, \mathrm{X}=-\mathrm{CH}_{2} \mathrm{CH}_{2}-$

18b $\mathrm{R}=\mathrm{Me}, \mathrm{R}_{1}=\mathrm{SO}_{2} \mathrm{Me}, \mathrm{X}=-\mathrm{CH}_{2} \mathrm{CH}_{2} \mathrm{O}-$

19a $\mathrm{R}=\mathrm{H}, \mathrm{R}_{1}=\mathrm{SO}_{2} \mathrm{Me}, \mathrm{X}=-\mathrm{CH}_{2} \mathrm{CH}_{2}-$

19b $\mathrm{R}=\mathrm{H}, \mathrm{R}_{1}=\mathrm{SO}_{2} \mathrm{Me}, \mathrm{X}=-\mathrm{CH}_{2} \mathrm{CH}_{2} \mathrm{O}-$

Scheme 3. Reagents and conditions: (a) $\mathrm{CH}_{3} \mathrm{NO}_{2}, \mathrm{CH}_{3} \mathrm{COONH}_{4}, 100{ }^{\circ} \mathrm{C}$; (b) $\mathrm{LiAlH}_{4}$, THF; (c) 4-nitrophenethyl or 2-(4-nitrophenoxy)ethylbromide, $\mathrm{K}_{2} \mathrm{CO}_{3}$, TBAI, anhyd $\mathrm{CH}_{3} \mathrm{CN}$; (d) $\mathrm{HCHO} 36 \%, \mathrm{HCOOH}, 100{ }^{\circ} \mathrm{C}$; (e) $\mathrm{NaBH}_{4}, \mathrm{CuCl}, \mathrm{EtOH}, 80^{\circ} \mathrm{C}$; (f) $\mathrm{CH}_{3} \mathrm{SO}_{2} \mathrm{Cl}_{2}, \mathrm{pyridine}$, anhyd $\mathrm{CH}_{2} \mathrm{Cl}_{2}$; (g) $\mathrm{BF}_{3} \cdot \mathrm{S}\left(\mathrm{CH}_{3}\right)_{2}$, anhyd $\mathrm{CH}_{2} \mathrm{Cl}_{2}$.

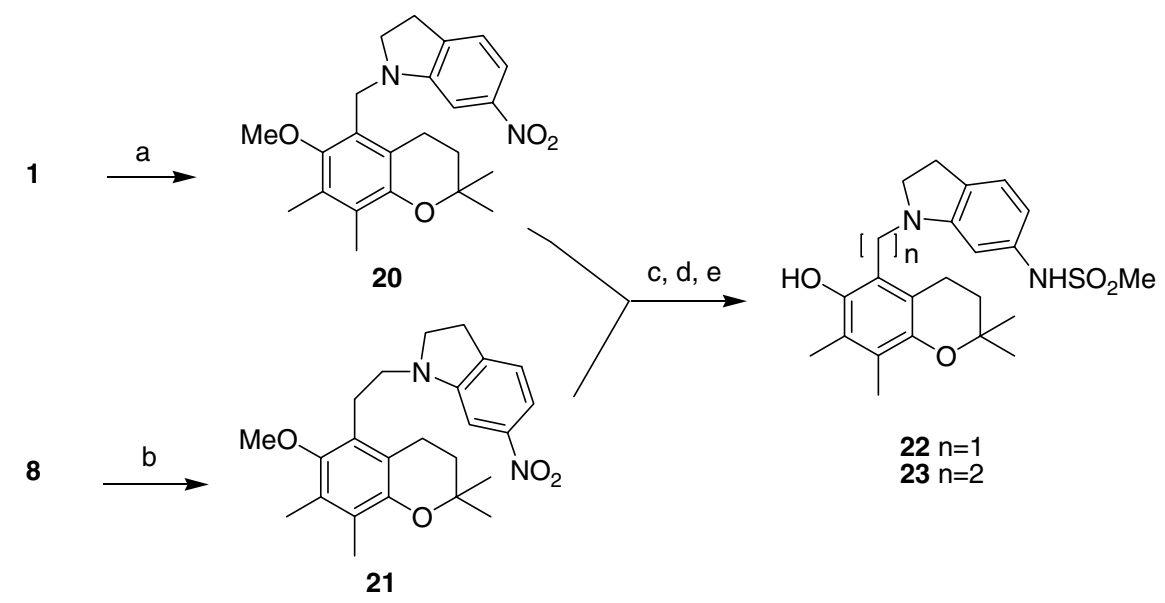

Scheme 4. Reagents and conditions: (a) 6-nitroindoline, $\mathrm{K}_{2} \mathrm{CO}_{3}$, TBAI, $\mathrm{CH}_{3} \mathrm{CN}$; (b) 6-nitroindoline, $\mathrm{NaBH}_{3} \mathrm{CN}_{2} \mathrm{CH}_{3} \mathrm{COOH}_{2} \mathrm{CH}_{3} \mathrm{CN}$; (c) $\mathrm{NaBH}$, $\mathrm{CuCl}$, EtOH, $80^{\circ} \mathrm{C}$; (d) $\mathrm{CH}_{3} \mathrm{SO}_{2} \mathrm{Cl}$, pyridine, anhyd $\mathrm{CH}_{2} \mathrm{Cl}_{2}$; (e) $\left.\mathrm{BF}_{3} \cdot \mathrm{S}_{(} \mathrm{CH}_{3}\right)_{2}$, anhyd $\mathrm{CH}_{2} \mathrm{Cl}_{2}$.

Table 1. Antiarrhythmic and antioxidant activity, at a concentration of $10 \mu \mathrm{M}$, of the new compounds

\begin{tabular}{lccl}
\hline Compound & Premature beats $(\%)$ & MDA (nmol/g) & Remarks \\
\hline None (control) & $3 \pm 0.6$ & $134 \pm 11$ & Tachycardia \\
$\mathbf{5 a}$ & $3 \pm 0.28$ & $137 \pm 13$ & Bradycardia \\
$\mathbf{5 b}$ & $3.15 \pm 1.06$ & $141 \pm 9$ & Tachycardia \\
$\mathbf{5 c}$ & $3.15 \pm 1.9$ & $146 \pm 25$ & Bradycardia \\
$\mathbf{5 d}$ & $8.2 \pm 1.8$ & $129 \pm 35$ & Increase of premature beats \\
$\mathbf{5 e}$ & $2.8 \pm 1.04$ & $95 \pm 35$ & Bradycardia \\
$\mathbf{1 2}$ & $5.35 \pm 2.1$ & $139 \pm 7$ & Fibrillation \\
$\mathbf{1 8 a}$ & $3.9 \pm 1.68$ & $105 \pm 7$ & Suppression of tachycardia \\
$\mathbf{1 9 a}$ & $1.35 \pm 0.58^{*}$ & $76 \pm 15^{* *}$ & Fast recovery of the heart \\
$\mathbf{1 9 b}$ & $3.05 \pm 1.06$ & $110 \pm 14$ & Suppression of tachycardia \\
$\mathbf{2 2}$ & $3.85 \pm 1.4$ & $104.5 \pm 16$ & Fast recovery \\
$\mathbf{2 3}$ & $2.11 \pm 0.75$ & $83 \pm 2.8^{* *}$ & Fast recovery \\
Amiodarone & $5.11 \pm 3.5$ & $116 \pm 5.6$ & Fibrillation \\
\hline
\end{tabular}

$n=3-4$.

${ }^{*} p<0.05$, versus control.

$p<0.01$, versus control. 
expressed as MDA content (nmoles/gr of cardiac tissue) at the end of reperfusion.

In the absence of compound (control) tachycardia was observed during reperfusion, while the QT and QRS intervals shorten during ischemia and reperfusion (Table 2).

Compound $\mathbf{5 b}$, which induces tachycardia upon reperfusion, and compounds $\mathbf{5 d}$ and $\mathbf{1 2}$, which increase the incidence of premature beats, are the less active analogues.

Piperazine analogue 5a suppresses tachycardia during reperfusion, prolongs QT interval during ischemia and reperfusion, while it affects QRS interval only during ischemia. Compound 5c suppresses also reperfusion tachycardia, prolongs QT interval during ischemia and reperfusion, and prolongs QRS interval only during reperfusion. Compound $\mathbf{5 e}$ is the only piperazine derivative that reduces premature beats and exhibits antioxidant activity.

Among the methylamino analogues, compound 19b, does not reduce the premature beats but suppresses reperfusion tachycardia. Although this analogue does not influence the QT interval during ischemia, it causes a significant QT widening during reperfusion and it slightly decreases MDA content. Compound 19a, bearing ethylene group instead of ethylenoxy group of compound $19 \mathrm{~b}$, and reduces premature beats, prolongs QT and QRS intervals during ischemia and reperfusion, reduces MDA content, leading to a fast recovery of the heart. In addition, analogue 19a exhibits better antiarrhythmic and antioxidant activity than its methoxy derivative $\mathbf{1 8 a}$.

The two constrained analogues $\mathbf{2 2}$ and $\mathbf{2 3}$ induced fast recovery of the heart during reperfusion. It should be also noted that the presence of compounds 5c, 19a,, $\mathbf{b}$, and 22 facilitated the recovery of QRS and QT intervals to the normal values.

The effect of compounds $\mathbf{5 a - e}$ and $\mathbf{1 9 a}, \mathbf{b}$ on the action potential parameters was investigated at $5 \mu \mathrm{M}$, in rab- bit ventricular muscles. The results are summarized in Table 3. The compounds did not change the resting potential (RP), the action potential amplitude (APA), and the maximal rate of depolarization $\left(V_{\max }\right)$. The observed variations of $V_{\max }$ in some experiments most likely reflect inconsistency of the impalement of the microelectrode. These data suggest no major change of the fast inward sodium current $\left(I_{\mathrm{Na}}\right)$ after the application of the analogues. Compounds 5a,b,d did not alter the repolarization process reflected as no change of the $50 \%$ and $90 \%$ action potential durations $\left(\mathrm{APD}_{50}\right.$ and $\left.\mathrm{APD}_{90}\right)$. This indicates no, or minimal effect of the major repolarizing potassium channels. However, compounds $5 \mathbf{c}, \mathbf{e}$ and 19a,b prolonged APD by $5-14 \%$ which suggests moderate inhibition of the repolarizing potassium current most likely of the rapid delayed rectifier potassium current $\left(I_{\mathrm{Kr}}\right.$ or HERG). The latter effect represents moderate class III antiarrhythmic actions and may need further investigations.

\section{Conclusions}

Among piperazine derivatives, compounds $\mathbf{5 c}$ and 5e suppress reperfusion tachycardia, while compound 5e reduces premature beats and MDA content, combining antiarrhythmic and antioxidant properties. The presence of phenyl piperazine moiety (analogues 5d and 12) abolishes antiarrhythmic activity.

Methylamino derivative 19a and its constrained analogue 23 exhibit antioxidant activity, reduce premature beats, and induce a fast recovery of the heart during reperfusion.

Compounds $\mathbf{5 c}, \mathbf{1 9 a}, \mathbf{b}$, and $\mathbf{2 2}$ facilitated the recovery of QRS and QT intervals during reperfusion, to the normal values. Moreover, the cardioprotective compounds $\mathbf{5 c}, \mathbf{e}$ and 19a,b do not induce excessive lengthening of the action potential, exhibiting moderate class III antiarrhythmic actions. Further studies on animal models as well as on the possible influence on specific potassium channels such as $I_{\mathrm{Kr}}, I_{\mathrm{Ks}}, I_{\mathrm{to}}$, and $I_{\mathrm{K} 1}$ should clarify the mecha-

Table 2. QRS and QT intervals (ms) of the analogues 5a,e,c, 19a,b, 22, and $\mathbf{2 3}$ during ischemia and reperfusion

\begin{tabular}{|c|c|c|c|c|c|c|}
\hline \multirow[t]{2}{*}{ Compound } & \multicolumn{2}{|c|}{ Equilibration } & \multicolumn{2}{|c|}{ Ischemia } & \multicolumn{2}{|c|}{ Reperfusion } \\
\hline & QRS & QT & QRS & QT & QRS & QT \\
\hline None (control) & $39 \pm 3.2$ & $105 \pm 7.3$ & $28 \pm 3.6$ & $61.5 \pm 4.3$ & $24.4 \pm 2.1$ & $55.4 \pm 4.8$ \\
\hline 5 a & & & $36 \pm 3.1^{*}$ & $114 \pm 9.6^{* * *}$ & $26.4 \pm 2.2$ & $121.3 \pm 11.7^{* * *}$ \\
\hline $5 c$ & & & $31 \pm 2.4$ & $96 \pm 7.4^{* *}$ & $42.7 \pm 3.2^{* * *}$ & $119.7 \pm 12^{* * *}$ \\
\hline $5 e$ & & & $29.14 \pm 1$ & $97.4 \pm 8.4^{* *}$ & $41.7 \pm 4.3^{* * *}$ & $129.15 \pm 14.6^{* * *}$ \\
\hline $19 a$ & & & $38.5 \pm 4.2^{* *}$ & $89.3 \pm 6.8^{* *}$ & $30.6 \pm 2.7^{*}$ & $107.6 \pm 10.3^{* * *}$ \\
\hline $19 b$ & & & $21.3 \pm 4$ & $63.3 \pm 9.3$ & $43.4 \pm 1.4^{* * *}$ & $121.3 \pm 5.7^{* * *}$ \\
\hline 22 & & & $32.5 \pm 4.4$ & $108.1 \pm 12.5^{* *}$ & $33.12 \pm 4.7^{*}$ & $106.16 \pm 24.7^{* *}$ \\
\hline 23 & & & $33.8 \pm 2.1$ & $102.8 \pm 3.6^{* * *}$ & $39.44 \pm 1.7^{* * *}$ & $143.44 \pm 9.2^{* * *}$ \\
\hline Amiodarone & & & $36.3 \pm 3.7^{* *}$ & $129.1 \pm 18.4^{* * *}$ & $42.4 \pm 2.3^{* * *}$ & $134.1 \pm 17.2^{* * *}$ \\
\hline
\end{tabular}

$n=3-4$.

${ }^{*} p<0.05$, versus control.

${ }^{* * *} p<0.01$, versus control.

${ }^{* *} p<0.001$, versus control. 
Table 3. Effect of analogues $\mathbf{5 a - e}$ and $\mathbf{1 9 a}, \mathbf{b}$, at a concentration of $5 \mu \mathrm{M}$, on the action potential parameters in rabbit isolated right ventricular papillary muscle

\begin{tabular}{|c|c|c|c|c|c|c|}
\hline Compound & Experiments & $\mathrm{RP}$ & APA & $\mathrm{APD}_{50}$ & $\mathrm{APD}_{90}$ & $V_{\max }$ \\
\hline $\begin{array}{l}\text { Control } \\
\mathbf{5 a}\end{array}$ & 1 & $\begin{array}{l}-88 \\
-88\end{array}$ & $\begin{array}{l}122 \\
125\end{array}$ & $\begin{array}{l}164 \\
167\end{array}$ & $\begin{array}{l}201 \\
201(0 \%)\end{array}$ & $\begin{array}{l}201 \\
201\end{array}$ \\
\hline $\begin{array}{l}\text { Control } \\
\mathbf{5 a}\end{array}$ & 2 & $\begin{array}{l}-89 \\
-87\end{array}$ & $\begin{array}{l}117 \\
115\end{array}$ & $\begin{array}{l}124 \\
133\end{array}$ & $\begin{array}{l}165 \\
173(4.8 \%)\end{array}$ & $\begin{array}{l}171 \\
186\end{array}$ \\
\hline $\begin{array}{l}\text { Control } \\
\mathbf{5 b}\end{array}$ & 1 & $\begin{array}{l}-84 \\
-85\end{array}$ & $\begin{array}{l}110 \\
118\end{array}$ & $\begin{array}{l}107 \\
110\end{array}$ & $\begin{array}{l}139 \\
140(0 \%)\end{array}$ & $\begin{array}{l}186 \\
208\end{array}$ \\
\hline $\begin{array}{l}\text { Control } \\
\mathbf{5 b}\end{array}$ & 2 & $\begin{array}{l}-93 \\
-91\end{array}$ & $\begin{array}{l}114 \\
115\end{array}$ & $\begin{array}{l}136 \\
136\end{array}$ & $\begin{array}{l}169 \\
168(0 \%)\end{array}$ & $\begin{array}{l}156 \\
163\end{array}$ \\
\hline $\begin{array}{l}\text { Control } \\
\mathbf{5 c}\end{array}$ & 1 & $\begin{array}{l}-87 \\
-86\end{array}$ & $\begin{array}{l}121 \\
122\end{array}$ & $\begin{array}{l}167 \\
188\end{array}$ & $\begin{array}{l}205 \\
224(9.3 \%)\end{array}$ & $\begin{array}{l}223 \\
230\end{array}$ \\
\hline $\begin{array}{l}\text { Control } \\
\mathbf{5 c}\end{array}$ & 2 & $\begin{array}{l}-77 \\
-84\end{array}$ & $\begin{array}{r}98 \\
109\end{array}$ & $\begin{array}{l}126 \\
159\end{array}$ & $\begin{array}{l}175 \\
199(13.7 \%)\end{array}$ & $\begin{array}{l}267 \\
267\end{array}$ \\
\hline $\begin{array}{l}\text { Control } \\
\mathbf{5 d}\end{array}$ & 1 & $\begin{array}{l}-90 \\
-88\end{array}$ & $\begin{array}{l}104 \\
100\end{array}$ & $\begin{array}{l}157 \\
157\end{array}$ & $\begin{array}{l}195 \\
189(-3.1 \%)\end{array}$ & $\begin{array}{l}171 \\
208\end{array}$ \\
\hline $\begin{array}{l}\text { Control } \\
\text { 5d }\end{array}$ & 2 & $\begin{array}{l}-89 \\
-89\end{array}$ & $\begin{array}{l}114 \\
112\end{array}$ & $\begin{array}{l}112 \\
137\end{array}$ & $\begin{array}{l}161 \\
169(5 \%)\end{array}$ & $\begin{array}{l}178 \\
216\end{array}$ \\
\hline $\begin{array}{l}\text { Control } \\
\mathbf{5 e}\end{array}$ & 1 & $\begin{array}{l}-84 \\
-84\end{array}$ & $\begin{array}{l}117 \\
117\end{array}$ & $\begin{array}{l}127 \\
150\end{array}$ & $\begin{array}{l}175 \\
191(9.1 \%)\end{array}$ & $\begin{array}{l}305 \\
297\end{array}$ \\
\hline $\begin{array}{l}\text { Control } \\
\mathbf{5 e}\end{array}$ & 2 & $\begin{array}{l}-93 \\
-92\end{array}$ & $\begin{array}{l}113 \\
112\end{array}$ & $\begin{array}{l}154 \\
167\end{array}$ & $\begin{array}{l}200 \\
211(5.5 \%)\end{array}$ & $\begin{array}{l}163 \\
171\end{array}$ \\
\hline $\begin{array}{l}\text { Control } \\
19 \mathbf{a}\end{array}$ & 1 & $\begin{array}{l}-90 \\
-91\end{array}$ & $\begin{array}{l}113 \\
120\end{array}$ & $\begin{array}{l}197 \\
214\end{array}$ & $\begin{array}{l}230 \\
246(6.9 \%)\end{array}$ & $\begin{array}{l}201 \\
230\end{array}$ \\
\hline $\begin{array}{l}\text { Control } \\
19 \mathbf{a}\end{array}$ & 2 & $\begin{array}{l}-82 \\
-86\end{array}$ & $\begin{array}{l}105 \\
106\end{array}$ & $\begin{array}{l}118 \\
127\end{array}$ & $\begin{array}{l}155 \\
170(9.7 \%)\end{array}$ & $\begin{array}{l}193 \\
260\end{array}$ \\
\hline $\begin{array}{l}\text { Control } \\
\text { 19b }\end{array}$ & 1 & $\begin{array}{l}-86 \\
-86\end{array}$ & $\begin{array}{l}119 \\
117\end{array}$ & $\begin{array}{l}110 \\
117\end{array}$ & $\begin{array}{l}150 \\
160(6.7 \%)\end{array}$ & $\begin{array}{l}171 \\
178\end{array}$ \\
\hline $\begin{array}{l}\text { Control } \\
\text { 19b }\end{array}$ & 2 & $\begin{array}{l}-86 \\
-83\end{array}$ & $\begin{array}{l}117 \\
103\end{array}$ & $\begin{array}{l}199 \\
215\end{array}$ & $\begin{array}{l}237 \\
255(7.6 \%)\end{array}$ & $\begin{array}{l}334 \\
201\end{array}$ \\
\hline
\end{tabular}

$\mathrm{RP}$, resting membrane potential.

APA, action potential amplitude.

$\mathrm{APD}_{50-90}=50 \%$ and $90 \%$ action potential duration.

$V_{\max }=$ maximal rate of depolarization.

nism and provide additional evidence of efficacy of these compounds.

\section{Experimental}

\subsection{Chemistry}

Melting points were determined on a Buchi 510 apparatus and are uncorrected. NMR spectra were recorded on a Bruker AC 300 spectrometer operating at $300 \mathrm{MHz}$ for ${ }^{1} \mathrm{H}$ and $75.43 \mathrm{MHz}$ for ${ }^{13} \mathrm{C}$. ${ }^{1} \mathrm{H}$ NMR spectra are reported in units of $\delta$ with $\mathrm{CHCl}_{3}$ resonance at $7.26 \mathrm{ppm}$ used as the chemical shift resonance. ${ }^{13} \mathrm{C}$ NMR spectra are reported in units of $\delta$ relative to $\mathrm{CDCl}_{3}$ at $77.00 \mathrm{ppm}$. $\mathrm{CDCl}_{3}$ was used as solvent. Silica gel plates MachereyNagel Sil G-25 UV 254 were used for thin-layer chromatography. Chromatographic purification was performed with silica gel (200-400 mesh). Mass spectra were recorded on a Varian Saturn 2000 GC-MS instrument in the EI mode. Elemental analyses were carried out on a PerkinElmer Series II CHNS/O 2400 analyser.

\subsection{General procedure for the synthesis of disubstituted piperazines $2 \mathrm{a}-\mathrm{e}(\operatorname{method} \mathrm{A})$}

To a solution of bromide $1(0.200 \mathrm{~g}, 0.64 \mathrm{mmol})$ in $5 \mathrm{~mL}$ anhyd $\mathrm{CH}_{3} \mathrm{CN}$, were added, at $0{ }^{\circ} \mathrm{C}, \mathrm{K}_{2} \mathrm{CO}_{3}$ $(0.132 \mathrm{~g}, 0.96 \mathrm{mmol})$ and a solution of the appropriate monosubstituted piperazine $(0.64 \mathrm{mmol})$ in $5 \mathrm{~mL}$ anhyd $\mathrm{CH}_{3} \mathrm{CN}$. A catalytic amount of TBAI was then added and the mixture was stirred at ambient temperature for $24 \mathrm{~h}$. After completion of the reaction, the solvent was evaporated, the residue was taken up with AcOEt and washed with water. The organic layer was dried and concentrated, and the residue was purified by column chromatography $\left(\mathrm{CH}_{2} \mathrm{Cl}_{2} / \mathrm{CH}_{3} \mathrm{OH} 9.5: 1.5\right)$.

5.2.1. 1-[(3,4-Dihydro-6-methoxy-2,2,7,8-tetramethyl2H-1-benzopyran-5-yl)methyl]-4-[(4-nitrophenyl)methyl]piperazine (2a). Yield: $85 \%$, yellow viscous oil. ${ }^{1} \mathrm{H}$ NMR $\delta: 8.14(\mathrm{~d}, 2 \mathrm{H}, J=9.1 \mathrm{~Hz}, \operatorname{Ar} H), 7.49(\mathrm{~d}$, $2 \mathrm{H}, J=9.1 \mathrm{~Hz}, \operatorname{Ar} H), 3.66\left(\mathrm{~s}, 3 \mathrm{H},-\mathrm{OCH}_{3}\right), 3.55(\mathrm{~s}$, $\left.2 \mathrm{H}, \quad \mathrm{ArCH}_{2} \mathrm{~N}-\right), 3.50$ (s, $\left.2 \mathrm{H},-\mathrm{NCH}_{2} \mathrm{Ar}\right), 2.86$ (t, $\left.2 \mathrm{H}, J=6.7 \mathrm{~Hz}, \mathrm{ArCH}_{2}\right), 2.51-2.49\left(\mathrm{~m}, 8 \mathrm{H}, \mathrm{CH}_{2}\right)$, 
$2.18\left(\mathrm{~s}, 3 \mathrm{H}, \mathrm{ArCH}_{3}\right), 2.10$ (s, 3H, $\left.\mathrm{ArCH}_{3}\right), 1.76$ (t, $\left.2 \mathrm{H}, J=6.7 \mathrm{~Hz}, \mathrm{CH}_{2}\right), 1.31\left(\mathrm{~s}, 6 \mathrm{H}, \mathrm{CH}_{3}\right){ }^{13} \mathrm{C} \mathrm{NMR}$ $\delta: 150.6,148.0,147.0,146.7,129.4,127.6,125.9$, $125.1,123.4,119.0,72.9,62.1,61.5,53.5,52.8,32.9$, 26.9, 20.1, 12.8, 12.1. MS $\mathrm{m} / z \quad 453\left(\mathrm{M}^{+} 1 \%\right), 268$ $(100 \%), 233$, 217. Anal. Calcd for $\mathrm{C}_{26} \mathrm{H}_{35} \mathrm{~N}_{3} \mathrm{O}_{4}: \mathrm{C}$, $68.85 ; \mathrm{H}, 7.78 ; \mathrm{N}, 9.26$. Found: C, 69.09; H, 7.88; $\mathrm{N}, 9.59$.

5.2.2. 1-I(3,4-Dihydro-6-methoxy-2,2,7,8-tetramethyl2H-1-benzopyran-5-yl)methyl]-4-[2-(4-nitrophenyl)ethyl]piperazine (2b). Yield: $80 \%$, yellow viscous oil. ${ }^{1} \mathrm{H}$ NMR $\delta: 8.09(\mathrm{~d}, 2 \mathrm{H}, J=9.1 \mathrm{~Hz}), 7.33(\mathrm{~d}, 2 \mathrm{H}, J=9.1 \mathrm{~Hz}), 3.63$ $(\mathrm{s}, 3 \mathrm{H}), 3.47(\mathrm{~s}, 3 \mathrm{H}), 2.84-2.82(\mathrm{~m}, 4 \mathrm{H}), 2.58(\mathrm{t}, 2 \mathrm{H}$, $J=6.7 \mathrm{~Hz}), 2.50-2.48(\mathrm{~m}, 8 \mathrm{H}), 2.16(\mathrm{~s}, 3 \mathrm{H}), 2.08(\mathrm{~s}$, $3 \mathrm{H}), 1.74(\mathrm{t}, 2 \mathrm{H}, J=6.7 \mathrm{~Hz}), 1.28(\mathrm{~s}, 6 \mathrm{H}) .{ }^{13} \mathrm{C} \mathrm{NMR}$ $\delta: 150.6,148.5,148.0,146.4,129.5,127.6,125.9,125.1$, $123.6,119.1,72.9,61.5,59.4,53.4,52.8,33.5,32.9$, $26.9,20.1,12.8,12.0$.

5.2.3. 1-[(3,4-Dihydro-6-methoxy-2,2,7,8-tetramethyl2H-1-benzopyran-5-yl)methyl|-4-[2-(4-nitrophenoxy)ethyl]piperazine (2c). Yield: $45 \%$, yellow viscous oil. ${ }^{1} \mathrm{H}$ NMR $\delta: 8.17(\mathrm{~d}, 2 \mathrm{H}, J=9.1 \mathrm{~Hz}), 6.92(\mathrm{~d}, 2 \mathrm{H}, J=9.1 \mathrm{~Hz}), 4.17$ $(\mathrm{t}, 2 \mathrm{H}, J=5.5 \mathrm{~Hz}), 3.64(\mathrm{~s}, 3 \mathrm{H}), 3.47(\mathrm{~s}, 2 \mathrm{H}), 2.84-2.79$ $(\mathrm{m}, 4 \mathrm{H}), 2.52-2.50(\mathrm{~m}, 8 \mathrm{H}), 2.17(\mathrm{~m}, 3 \mathrm{H}), 2.09(\mathrm{~s}, 3 \mathrm{H})$, $1.75(\mathrm{t}, 2 \mathrm{H}, J=6.7 \mathrm{~Hz}), 1.29(\mathrm{~s}, 6 \mathrm{H}) .{ }^{13} \mathrm{C}$ NMR $\delta$ : $163.8,150.5,148.0,141.5,127.6,125.8,125.1,119.1$, $114.5,114.4,72.9,66.9,65.0,61.5,56.8,54.0,53.1$, 52.7, 32.9, 26.9, 20.1, 12.8, 12.0. Anal. Calcd for $\mathrm{C}_{27} \mathrm{H}_{37} \mathrm{~N}_{3} \mathrm{O}_{5}$ : C, 67.06; H, 7.71; N, 8.69. Found: $\mathrm{C}$, $67.12 ; \mathrm{H}, 7.63 ; \mathrm{N}, 8.32$.

5.2.4. 1-[(3,4-Dihydro-6-methoxy-2,2,7,8-tetramethyl2H-1-benzopyran-5-yl)methyl]-4-(4-nitrophenyl)piperazine (2d). Bromide $\mathbf{1}$ and 1-(4-nitrophenyl)piperazine $(0.100 \mathrm{~g}, 0.48 \mathrm{mmol})$, in anhyd DMF at $80^{\circ} \mathrm{C}$, were treated according to method A. Yield: $0.154 \mathrm{~g}(73 \%)$, orange solid, mp $126-129^{\circ} \mathrm{C} .{ }^{1} \mathrm{H}$ NMR $\delta: 8.09$ (d, $2 \mathrm{H}, J=9.1 \mathrm{~Hz}), 6.78(\mathrm{~d}, 2 \mathrm{H}, J=9.1 \mathrm{~Hz}), 3.64(\mathrm{~s}$, $3 \mathrm{H}), 3.52(\mathrm{~s}, 2 \mathrm{H}), 3.36-3.34(\mathrm{~m}, 4 \mathrm{H}), 2.87(\mathrm{t}, 2 \mathrm{H}$, $J=6.7 \mathrm{~Hz}), 2.59-2.57(\mathrm{~m}, 4 \mathrm{H}), 2.19(\mathrm{~s}, 3 \mathrm{H}), 2.10(\mathrm{~s}$, $3 \mathrm{H}), 1.76(\mathrm{t}, 2 \mathrm{H}, J=6.7 \mathrm{~Hz}), 1.30(\mathrm{~s}, 6 \mathrm{H}) \cdot{ }^{13} \mathrm{C} \mathrm{NMR}$ $\delta: 154.9,150.5,127.8,125.9,125.2,119.0,112.4,73.0$, 61.6, 52.3, 47.2, 32.8, 26.9, 20.1, 12.8, 12.1. MS m/z: $234(100 \%)$, 219. Anal. Calcd for $\mathrm{C}_{25} \mathrm{H}_{33} \mathrm{~N}_{3} \mathrm{O}_{4}: \mathrm{C}$, 68.31; H, 7.57; N, 9.56. Found: C, 67.92; H, 7.62; N, 9.16 .

5.2.5. 1-[(3,4-Dihydro-6-methoxy-2,2,7,8-tetramethyl2H-1-benzopyran-5-yl)methyl]-4-(4-nitrobenzoyl)piperazine (2e). Yield: $95 \%$, yellow solid, mp $192-194{ }^{\circ} \mathrm{C} .{ }^{1} \mathrm{H}$ NMR $\delta: 8.20(\mathrm{~d}, 2 \mathrm{H}, J=8.5 \mathrm{~Hz}), 7.52(\mathrm{~d}, 2 \mathrm{H}$, $J=8.5 \mathrm{~Hz}), 3.74-3.72(\mathrm{~m}, 2 \mathrm{H}), 3.59(\mathrm{~s}, 3 \mathrm{H}), 3.51(\mathrm{~s}$, $2 \mathrm{H}), \quad 3.25-3.23(\mathrm{~m}, 2 \mathrm{H}), 2.81(\mathrm{t}, 2 \mathrm{H}, J=6.7 \mathrm{~Hz})$, $2.59-2.57(\mathrm{~m}, 2 \mathrm{H}), 2.39-2.37(\mathrm{~m}, 2 \mathrm{H}), 2.12(\mathrm{~s}, 3 \mathrm{H})$, $2.05(\mathrm{~s}, 3 \mathrm{H}), 1.74(\mathrm{t}, 2 \mathrm{H}, J=6.7 \mathrm{~Hz}), 1.25(\mathrm{~s}, 6 \mathrm{H})$. ${ }^{13} \mathrm{C}$ NMR $\delta: 167.8,150.5,148.2,142.2,128.0$, $127.7,125.6,125.0,123.8,118.9,73.0,65.0,61.5$, $52.7,47.9,42.5,32.8,26.9,20.0,12.7,12.0$. MS m/ $z: 234(100 \%), 219$. Anal. Calcd for $\mathrm{C}_{26} \mathrm{H}_{33} \mathrm{~N}_{3} \mathrm{O}_{5}$ : C, 66.79; H, 7.11; N, 8.99. Found: C, 66.86; H, 7.05; $\mathrm{N}, 8.67$.
5.3. General procedure for the synthesis of 4-substituted anilines (method B)

To a solution of the appropriate disubstituted piperazine $(0.35 \mathrm{mmol})$ in $8 \mathrm{~mL}$ abs $\mathrm{EtOH}, \mathrm{CuCl}(1.56 \mathrm{mmol})$ was added, at $0{ }^{\circ} \mathrm{C}$, followed by $\mathrm{NaBH}_{4}(3.18 \mathrm{mmol})$ and the mixture was refluxed for $2 \mathrm{~h}$. The mixture was then filtered through Celite and washed with $\mathrm{CH}_{2} \mathrm{Cl}_{2}$. The filtrate was washed with sat aqueous $\mathrm{NaCl}$, dried, and evaporated to dryness.

5.3.1. [4-[4-[(3,4-Dihydro-6-methoxy-2,2,7,8-tetramethyl2H-1-benzopyran-5-yl)methyl]piperazin-1-yl]methyl]aniline (3a). Yield: 95\%, yellow viscous oil. ${ }^{1} \mathrm{H}$ NMR $\delta$ : $7.09(\mathrm{~d}, 2 \mathrm{H}, J=8.5 \mathrm{~Hz}), 6.61(\mathrm{~d}, 2 \mathrm{H}, J=8.5 \mathrm{~Hz}), 3.63$ $(\mathrm{s}, 3 \mathrm{H}), 3.47(\mathrm{~s}, 2 \mathrm{H}), 3.41(\mathrm{~s}, 2 \mathrm{H}), 2.81(\mathrm{t}, 2 \mathrm{H}$, $J=6.7 \mathrm{~Hz}), 2.49-2.47(\mathrm{~m}, 8 \mathrm{H}), 2.16(\mathrm{~s}, 3 \mathrm{H}), 2.08(\mathrm{~s}$, $3 \mathrm{H}), 1.73(\mathrm{t}, 2 \mathrm{H}, J=6.7 \mathrm{~Hz}), 1.28(\mathrm{~s}, 6 \mathrm{H})$.

5.3.2. [4-[4-[(3,4-Dihydro-6-methoxy-2,2,7,8-tetramethyl2H-1- benzopyran-5-yl)methyl]piperazin-1-yl]ethyl]aniline (3b). Yield: 88\%, yellow viscous oil. ${ }^{1} \mathrm{H}$ NMR $\delta: 7.05(\mathrm{~d}$, $2 \mathrm{H}, J=9.1 \mathrm{~Hz}), 6.63(\mathrm{~d}, 2 \mathrm{H}, J=9.1 \mathrm{~Hz}), 3.66(\mathrm{~s}, 3 \mathrm{H})$, $3.47(\mathrm{~s}, \quad 3 \mathrm{H}), 2.85-2.82(\mathrm{~m}, 4 \mathrm{H}), 2.59(\mathrm{t}, \quad 2 \mathrm{H}$, $J=6.7 \mathrm{~Hz}), 2.51-2.48(\mathrm{~m}, 8 \mathrm{H}), 2.17(\mathrm{~s}, 3 \mathrm{H}), 2.08(\mathrm{~s}$, $3 \mathrm{H}), 1.74(\mathrm{t}, 2 \mathrm{H}, J=6.7 \mathrm{~Hz}), 1.27(\mathrm{~s}, 6 \mathrm{H})$.

5.3.3. 4-[2-[4-[(3,4-Dihydro-6-methoxy-2,2,7,8-tetramethyl-2H-1-benzopyran-5-yl)methyl]piperazin-1-yl]ethoxylaniline (3c). Yield: $80 \%$, yellow viscous oil. ${ }^{1} \mathrm{H}$ NMR $\delta$ : $6.71(\mathrm{~d}, 2 \mathrm{H}, J=8.5 \mathrm{~Hz}), 6.61(\mathrm{~d}, 2 \mathrm{H}, J=8.5 \mathrm{~Hz}), 4.05(\mathrm{t}$, $2 \mathrm{H}, J=5.5 \mathrm{~Hz}), 3.64(\mathrm{~s}, 3 \mathrm{H}), 3.47(\mathrm{~s}, 2 \mathrm{H}), 2.84(\mathrm{t}, 2 \mathrm{H}$, $J=6.7 \mathrm{~Hz}), 2.75(\mathrm{t}, 2 \mathrm{H}, J=5.5 \mathrm{~Hz}), 2.52-2.50(\mathrm{~m}$, $8 \mathrm{H}), 2.16(\mathrm{~s}, 3 \mathrm{H}), 2.08(\mathrm{~s}, 3 \mathrm{H}), \quad 1.74 \quad(\mathrm{t}, 2 \mathrm{H}$, $J=6.7 \mathrm{~Hz}), 1.28(\mathrm{~s}, 6 \mathrm{H})$.

5.3.4. 4-[4-[(3,4-Dihydro-6-methoxy-2,2,7,8-tetramethyl2H-1-benzopyran-5-yl)methyl|piperazin-1-yl]aniline (3d). Yield: $0.137 \mathrm{~g}(96 \%)$, green viscous oil. ${ }^{1} \mathrm{H}$ NMR $\delta: 6.78$ $(\mathrm{d}, 2 \mathrm{H}, J=8.5 \mathrm{~Hz}), 6.63(\mathrm{~d}, 2 \mathrm{H}, J=8.5 \mathrm{~Hz}), 3.66(\mathrm{~s}$, $3 \mathrm{H}), 3.55(\mathrm{~s}, 2 \mathrm{H}), 2.99-2.97(\mathrm{~m}, 4 \mathrm{H}), 2.87(\mathrm{t}, 2 \mathrm{H}$, $J=6.7 \mathrm{~Hz}), 2.63-2.61(\mathrm{~m}, 4 \mathrm{H}), 2.19(\mathrm{~s}, 3 \mathrm{H}), 2.10(\mathrm{~s}$, $3 \mathrm{H}), 1.75(\mathrm{t}, 2 \mathrm{H}, J=6.7 \mathrm{~Hz}), 1.29(\mathrm{~s}, 6 \mathrm{H})$.

5.3.5. 4-[|4-[(3,4-Dihydro-6-methoxy-2,2,7,8-tetramethyl2H-1-benzopyran-5-yl)methyl]piperazin-1-yl]carbonyl]aniline (3e). Yield: $78 \%$, yellow viscous oil. ${ }^{1} \mathrm{H}$ NMR $\delta$ : 7.23 $(\mathrm{d}, 2 \mathrm{H}, J=8.5 \mathrm{~Hz}), 6.61(\mathrm{~d}, 2 \mathrm{H}, J=8.5 \mathrm{~Hz}), 3.84$ $3.82(\mathrm{~m}, 2 \mathrm{H}), 3.63(\mathrm{~s}, 3 \mathrm{H}), 3.48-3.47(\mathrm{~m}, 2 \mathrm{H})$, $2.84(\mathrm{t}, 2 \mathrm{H}, J=6.7 \mathrm{~Hz}), 2.44-2.42(\mathrm{~m}, 4 \mathrm{H}), 2.16(\mathrm{~s}$, $3 \mathrm{H}), 2.08(\mathrm{~s}, 3 \mathrm{H}), 1.75(\mathrm{t}, 2 \mathrm{H}, J=6.7 \mathrm{~Hz}), 1.28(\mathrm{~s}$, $6 \mathrm{H})$.

5.4. General procedure for the synthesis of 4-substituted phenylmethanesulfonamides (method C)

To a solution of the appropriate aniline $(0.5 \mathrm{mmol})$ in $6 \mathrm{~mL} \mathrm{CH}_{2} \mathrm{Cl}_{2}$ and $2 \mathrm{~mL}$ pyridine was added at $0{ }^{\circ} \mathrm{C}$ $\mathrm{CH}_{3} \mathrm{SO}_{2} \mathrm{Cl}(1 \mathrm{mmol})$ and the mixture was stirred at ambient temperature. After completion of the reaction, AcOEt and sat aqueous solution of $\mathrm{NH}_{4} \mathrm{Cl}$ were added. The organic layer was further washed by satd aqueous $\mathrm{NaCl}$, dried, and concentrated in vacuo. The residue 
was purified by column chromatography $\left(\mathrm{CH}_{2} \mathrm{Cl}_{2} /\right.$ $\mathrm{CH}_{3} \mathrm{OH}$ 9.5:1.5).

5.4.1. $\quad N$-[4-[[4-[(3,4-Dihydro-6-methoxy-2,2,7,8-tetramethyl-2H-1-benzopyran-5-yl)methyl]piperazin-1-yl]methyl]phenyl|methanesulfonamide (4a). Yield: $55 \%$, white solid, mp 80-82 ${ }^{\circ} \mathrm{C} .{ }^{1} \mathrm{H}$ NMR $\delta: 7.27(\mathrm{~d}, 2 \mathrm{H}, J=8.5 \mathrm{~Hz}), 7.15$ $(\mathrm{d}, 2 \mathrm{H}, J=8.5 \mathrm{~Hz}), 3.64(\mathrm{~s}, 3 \mathrm{H}), 3.47(\mathrm{~s}, 2 \mathrm{H}), 3.42(\mathrm{~s}$, $2 \mathrm{H}), 2.98(\mathrm{~s}, 3 \mathrm{H}), 2.83(\mathrm{t}, 2 \mathrm{H}, J=6.7 \mathrm{~Hz}), 2.47-2.38$ $(\mathrm{m}, 8 \mathrm{H}), 2.16(\mathrm{~s}, 3 \mathrm{H}), 2.08(\mathrm{~s}, 3 \mathrm{H}), 1.74(\mathrm{t}, 2 \mathrm{H}$, $J=6.7 \mathrm{~Hz}), 1.28(\mathrm{~s}, 6 \mathrm{H}) .{ }^{13} \mathrm{C}$ NMR $\delta: 150.5,148.0$, $135.5,130.3,127.6,125.9,125.0,120.9,119.1,72.9$, $62.2,61.5,53.4,52.8,39.2,32.9,26.9,20.1,12.8,12.0$. MS $m / z: 234(100 \%), 219,179$. Anal. Calcd for $\mathrm{C}_{27} \mathrm{H}_{39} \mathrm{~N}_{3} \mathrm{O}_{4} \mathrm{~S}$ : C, 64.64; H, 7.84; N, 8.38. Found: $\mathrm{C}$, 64.36; H, 7.56; N, 8.16.

5.4.2. $\quad N$-[4-[2-[4-[(3,4-Dihydro-6-methoxy-2,2,7,8-tetramethyl- 2H-1-benzopyran-5-yl)methyl]piperazin-1-yl]ethyl|phenyl|methanesulfonamide (4b). Yield $80 \%$, white solid, mp $152-155^{\circ} \mathrm{C} .{ }^{1} \mathrm{H}$ NMR $\delta: 7.15-7.13(\mathrm{~m}, 4 \mathrm{H})$, $3.65(\mathrm{~s}, 3 \mathrm{H}), 3.48(\mathrm{~s}, 2 \mathrm{H}), 2.96\left(\mathrm{~s}, 3 \mathrm{H},-\mathrm{NHSO}_{2} \mathrm{CH}_{3}\right)$, $2.84(\mathrm{t}, 2 \mathrm{H}, J=6.7 \mathrm{~Hz}), 2.75-2.70(\mathrm{~m}, 2 \mathrm{H}), 2.53-2.51$ $(\mathrm{m}, 10 \mathrm{H}),, 2.17(\mathrm{~s}, 3 \mathrm{H}), 2.09(\mathrm{~s}, 3 \mathrm{H}), 1.75(\mathrm{t}, 2 \mathrm{H}$, $J=6.7 \mathrm{~Hz}), 1.29(\mathrm{~s}, 6 \mathrm{H}) .{ }^{13} \mathrm{C}$ NMR $\delta: 150.5,148.0$, 137.9 , 134.7, 129.8, 127.6, 125.9, 125.1, 121.6, 119.1, $72.9,61.5,60.2,53.4,52.8,39.1,32.9,26.9,20.1,12.8$, 12.1. MS $m / z$ : 461, $234(100 \%), 219,179$. Anal. Calcd for $\mathrm{C}_{28} \mathrm{H}_{41} \mathrm{~N}_{3} \mathrm{O}_{4} \mathrm{~S}$ : C, 65.21; H, 8.01; N, 8.15. Found: C, 64.82; H, 8.35; N, 7.73.

5.4.3. $\quad N-[4-[2-[4-[(3,4-D i h y d r o-6-m e t h o x y-2,2,7,8$-tetramethyl-2H-1-benzopyran-5-yl)methyl]piperazin-1-yl]ethoxylphenyl|methanesulfonamide (4c). Yield: $74 \%$, white viscous oil. ${ }^{1} \mathrm{H}$ NMR $\delta: 7.17(\mathrm{~d}, 2 \mathrm{H}, J=8.5 \mathrm{~Hz}), 6.83$ $(\mathrm{d}, 2 \mathrm{H}, J=8.5 \mathrm{~Hz}), 4.10(\mathrm{t}, 2 \mathrm{H}, J=5.5 \mathrm{~Hz}), 3.62(\mathrm{~s}$, $3 \mathrm{H}), 3.47(\mathrm{~s}, 2 \mathrm{H}), 2.91(\mathrm{~s}, 3 \mathrm{H}), 2.83(\mathrm{t}, 2 \mathrm{H}, J=6.7 \mathrm{~Hz})$, $2.77(\mathrm{t}, 2 \mathrm{H}, J=5.5 \mathrm{~Hz}), 2.52-2.50(\mathrm{~m}, 8 \mathrm{H}), 2.16(\mathrm{~s}, 3 \mathrm{H})$, $2.08(\mathrm{~s}, 3 \mathrm{H}), 1.73(\mathrm{t}, 2 \mathrm{H}, J=6.7 \mathrm{~Hz}), 1.28(\mathrm{~s}, 6 \mathrm{H}) .{ }^{13} \mathrm{C}$ NMR $\delta: 160.0,150.7,129.4,124.7,119.1,115.5,73.1$, $65.9,65.0,61.4,56.8,53.4,52.7,52.1,38.9,32.8,26.9$, 20.2, 12.8, 12.1. Anal. Calcd for $\mathrm{C}_{28} \mathrm{H}_{41} \mathrm{~N}_{3} \mathrm{O}_{5} \mathrm{~S} \cdot \mathrm{H}_{2} \mathrm{O}: \mathrm{C}$, 61.18; H, 7.88; N, 7.64. Found: C, 61.27; H, 7.58; N, 7.53.

5.4.4. $N$-[4-[4-[(3,4-Dihydro-6-methoxy-2,2,7,8-tetramethyl-2H-1-benzopyran-5-yl)methyl]piperazin-1-yl]phenyl]methanesulfonamide (4d). Yield: $82 \%$, white viscous oil. ${ }^{1} \mathrm{H}$ NMR $\delta: 7.13(\mathrm{~d}, 2 \mathrm{H}, J=9.1 \mathrm{~Hz}), 6.85(\mathrm{~d}, 2 \mathrm{H}$, $J=9.1 \mathrm{~Hz}), 3.66(\mathrm{~s}, 3 \mathrm{H}), 3.53(\mathrm{~s}, 2 \mathrm{H}), 3.10-3.08(\mathrm{~m}, 4 \mathrm{H})$, $2.91(\mathrm{~s}, 3 \mathrm{H}), 2.87(\mathrm{t}, 2 \mathrm{H}, J=6.7 \mathrm{~Hz}), 2.60-2.58(\mathrm{~m}, 4 \mathrm{H})$, $2.19(\mathrm{~s}, 3 \mathrm{H}), 2.10(\mathrm{~s}, 3 \mathrm{H}), 1.77(\mathrm{t}, 2 \mathrm{H}, J=6.7 \mathrm{~Hz}), 1.29$ $(\mathrm{s}, 6 \mathrm{H}) .{ }^{13} \mathrm{C}$ NMR $\delta: 150.5,150.0,148.1,127.6,125.3$, $124.5,119.1,116.5,73.0,61.6,52.7,49.2,38.7,32.9$, 26.9, 20.1, 12.8, 12.1. Anal. Calcd for $\mathrm{C}_{26} \mathrm{H}_{37} \mathrm{~N}_{3} \mathrm{O}_{4} \mathrm{~S}$ : C, 64.04; H, 7.65; N, 8.62. Found: C, 63.75; H, 7.73; N, 8.66.

5.4.5. $\quad N$-[4-[[4-[(3,4-Dihydro-6-methoxy-2,2,7,8-tetramethyl-2H-1-benzopyran-5-yl)methyl]piperazin-1-ylcarbonyl]phenyl]methanesulfonamide (4e). Yield: $50 \%$, yellowish solid, mp $194-196{ }^{\circ} \mathrm{C} .{ }^{1} \mathrm{H}$ NMR $\delta$ : 7.34 (d, $2 \mathrm{H}, J=8.5 \mathrm{~Hz}), 7.21(\mathrm{~d}, 2 \mathrm{H}, J=8.5 \mathrm{~Hz}), 3.67-3.65$ $(\mathrm{m}, 2 \mathrm{H}), 3.62(\mathrm{~s}, 3 \mathrm{H}), 3.49(\mathrm{~s}, 2 \mathrm{H}), 3.33-3.31(\mathrm{~m}, 2 \mathrm{H})$ $2.97(\mathrm{~s}, 3 \mathrm{H}), 2.83(\mathrm{t}, 2 \mathrm{H}, J=6.7 \mathrm{~Hz}), 2.52-2.50(\mathrm{~m}$,
$2 \mathrm{H}), 2.38-2.36(\mathrm{~m}, 2 \mathrm{H}), 2.15(\mathrm{~s}, 3 \mathrm{H}), 2.08(\mathrm{~s}, 3 \mathrm{H}), 1.75$ $(\mathrm{t}, 2 \mathrm{H}, J=6.7 \mathrm{~Hz}), 1.28(\mathrm{~s}, 6 \mathrm{H}) .{ }^{13} \mathrm{C}$ NMR $\delta: 169.8$, $150.6,148.2,139.0,131.4,128.7,127.8,119.6,73.1$, $61.5,52.9,52.6,50.6,39.4,32.8,29.7,26.9,20.1,12.8$, 12.1. Anal. Calcd for $\mathrm{C}_{27} \mathrm{H}_{37} \mathrm{~N}_{3} \mathrm{O}_{5} \mathrm{~S}$ : C, 62.89; H, 7.23; N, 8.15. Found: C, 62.65; H, 6.96; N, 8.14.

\subsection{General procedure for the synthesis of final phen- ylmethanesulfonamides (method D)}

To a solution of the appropriate phenylmethanesulfonamide $(0.1 \mathrm{mmol})$ in $4 \mathrm{~mL}$ anhyd $\mathrm{CH}_{2} \mathrm{Cl}_{2}$ was added, at $0{ }^{\circ} \mathrm{C}, \mathrm{BF}_{3} \cdot \mathrm{S}\left(\mathrm{CH}_{3}\right)_{2}(1 \mathrm{mmol})$ and the mixture was stirred at ambient temperature for $24 \mathrm{~h}$. The solvent is then evaporated under argon, and AcOEt and water were added. The organic layer was dried and evaporated to dryness. The residue was purified by column chromatography $\left(\mathrm{CH}_{2} \mathrm{Cl}_{2} / \mathrm{CH}_{3} \mathrm{OH}\right.$ 9.5:1.5).

5.5.1. $\quad N$-[4-[[4-[(3,4-Dihydro-6-hydroxy-2,2,7,8-tetramethyl-2H-1-benzopyran-5-yl)methyl|piperazin-1-yl|methyl]phenyl|methanesulfonamide (5a). Yield: $24 \%$, white solid, mp $212-215^{\circ} \mathrm{C} .{ }^{1} \mathrm{H}$ NMR $\delta: 7.30(\mathrm{~d}, 2 \mathrm{H}, J=8.5 \mathrm{~Hz})$, $7.17(\mathrm{~d}, 2 \mathrm{H}, J=8.5 \mathrm{~Hz}), 3.63(\mathrm{~s}, 2 \mathrm{H}), 3.50(\mathrm{~s}, 2 \mathrm{H})$, $3.00(\mathrm{~s}, 3 \mathrm{H}), 2.61-2.56(\mathrm{~m}, 10 \mathrm{H}), 2.12(\mathrm{~s}, 3 \mathrm{H}), 2.08(\mathrm{~s}$, $3 \mathrm{H}), 1.74(\mathrm{t}, 2 \mathrm{H}, J=6.7 \mathrm{~Hz}), 1.25(\mathrm{~s}, 6 \mathrm{H}) .{ }^{13} \mathrm{C} \mathrm{NMR}$ $\delta: 148.7,144.4,130.4,124.9,120.8,115.7,114.8,72.2$, $61.9,56.3,52.7,39.4,33.0,29.7,26.6,20.7,11.8,11.7$. MS $m / z: 487\left(\mathbf{M}^{+}\right), 438,257,219(100 \%)$. Anal. Calcd for $\mathrm{C}_{26} \mathrm{H}_{37} \mathrm{~N}_{3} \mathrm{O}_{4} \mathrm{~S}$ : C, 64.04; H, 7.65; N, 8.62. Found: C, 63.88; H, 7.35; N, 8.97.

5.5.2. $\quad N$-[4-[2-[4-[(3,4-Dihydro-6-hydroxy-2,2,7,8-tetramethyl-2H-1-benzopyran-5-yl)methyl]piperazin-1-yl]ethyl]phenyl|methanesulfonamide (5b). Yield: $41 \%$, white solid, mp $185-187^{\circ} \mathrm{C} .{ }^{1} \mathrm{H}$ NMR $\delta: 7.24$ (d, $2 \mathrm{H}$, $J=8.5 \mathrm{~Hz}), 7.07(\mathrm{~d}, 2 \mathrm{H}, J=8.5 \mathrm{~Hz}), 3.78(\mathrm{~s}, 2 \mathrm{H}), 3.61-$ $3.59(\mathrm{~m}, 2 \mathrm{H}), 3.15-3.13(\mathrm{~m}, 2 \mathrm{H}), 3.06-3.03(\mathrm{~m}, 8 \mathrm{H})$, $2.93(\mathrm{~s}, 3 \mathrm{H}), 2.58(\mathrm{t}, 2 \mathrm{H}, J=6.7 \mathrm{~Hz}), 2.08(\mathrm{~s}, 3 \mathrm{H}), 2.06$ $(\mathrm{s}, 3 \mathrm{H}), 1.72(\mathrm{t}, 2 \mathrm{H}, J=6.7 \mathrm{~Hz}), 1.24(\mathrm{~s}, 6 \mathrm{H}) \cdot{ }^{13} \mathrm{C} \mathrm{NMR}$ $\delta: 147.8,145.1,136.7,132.4,129.7,126.1,121.5,116.3$, $113.9,72.5,65.0,58.0,55.2,51.8,48.9,39.2,32.8,29.4$, 26.6, 20.8, 12.0, 11.9. Anal. Calcd for $\mathrm{C}_{27} \mathrm{H}_{39} \mathrm{~N}_{3} \mathrm{O}_{4} \mathrm{~S}: \mathrm{C}$, $64.64 ; \mathrm{H}, 7.84 ; \mathrm{N}, 8.38$. Found: $\mathrm{C}, 64.78 ; \mathrm{H}, 8.10 ; \mathrm{N}, 8.53$.

5.5.3. $\quad N$-[4-[2-[4-[(3,4-Dihydro-6-hydroxy-2,2,7,8-tetramethyl-2H-1-benzopyran-5-yl)methyl]piperazin-1-yl]ethoxylphenyl]methanesulfonamide (5c). Yield: $36 \%$, yellowish solid, mp $202-204{ }^{\circ} \mathrm{C} .{ }^{1} \mathrm{H}$ NMR $\delta: 7.17$ (d, $2 \mathrm{H}, J=9.1 \mathrm{~Hz}), 6.87(\mathrm{~d}, 2 \mathrm{H}, J=9.1 \mathrm{~Hz}), 4.09-4.07-$ $4.05(\mathrm{~m}, 2 \mathrm{H}), 3.65(\mathrm{~s}, 2 \mathrm{H}), 2.93(\mathrm{~s}, 3 \mathrm{H}), 2.89-2.86(\mathrm{~m}$, $2 \mathrm{H}), 2.71-2.69(\mathrm{~m}, 8 \mathrm{H}), 2.59(\mathrm{t}, 2 \mathrm{H}, J=6.7 \mathrm{~Hz}), 2.12$ $(\mathrm{s}, 3 \mathrm{H}), 2.09(\mathrm{~s}, 3 \mathrm{H}), 1.75(\mathrm{t}, 2 \mathrm{H}, J=6.7 \mathrm{~Hz}), 1.26(\mathrm{~s}$, $6 \mathrm{H}) .{ }^{13} \mathrm{C}$ NMR $\delta: 156.5,148.2,144.5,130.0,125.0$, $124.2,122.5,115.9,115.3,114.8,72.3,65.6,56.8,56.1$, 53.4, 53.2, 51.8, 38.5, 32.9, 26.5, 20.6, 11.8, 11.6. Anal. Calcd for $\mathrm{C}_{27} \mathrm{H}_{39} \mathrm{~N}_{3} \mathrm{O}_{5} \mathrm{~S}: \mathrm{C}, 62.64 ; \mathrm{H}, 7.59 ; \mathrm{N}, 8.12$. Found: C, 63.02; H, 7.97; N, 8.36.

5.5.4. $N$-[4-[4-[(3,4-Dihydro-6-hydroxy-2,2,7,8-tetramethyl-2H-1-benzopyran-5-yl)methyl]piperazin-1-yl]phenyl]methanesulfonamide (5d). Yield: 33\%, yellowish viscous oil. ${ }^{1} \mathrm{H}$ NMR $\delta: 7.16(\mathrm{~d}, 2 \mathrm{H}, J=8.5 \mathrm{~Hz}), 6.87(\mathrm{~d}, 2 \mathrm{H}$, 
$J=8.5 \mathrm{~Hz}), 3.72(\mathrm{~s}, 2 \mathrm{H}), 3.22-3.20(\mathrm{~m}, 4 \mathrm{H}) 2.93(\mathrm{~s}, 3 \mathrm{H})$, $2.70-2.68(\mathrm{~m}, 4 \mathrm{H}), 2.62(\mathrm{t}, 2 \mathrm{H}, J=6.7 \mathrm{~Hz}) 2.14(\mathrm{~s}, 3 \mathrm{H})$, $2.10(\mathrm{~s}, 3 \mathrm{H}), 1.77(\mathrm{t}, 2 \mathrm{H}, J=6.7 \mathrm{~Hz}), 1.28(\mathrm{~s}, 6 \mathrm{H}) .{ }^{13} \mathrm{C}$ NMR $\delta$ : 150.3, 148.9, 148.0, 126.9, 125.1, 124.0, 119.3, $115.8,72.8,62.1,52.5,38.8,32.9,26.6,20.8,11.9,11.6$. Anal. Calcd for $\mathrm{C}_{25} \mathrm{H}_{35} \mathrm{~N}_{3} \mathrm{O}_{4} \mathrm{~S}$ : C, 63.40; H, 7.45; N, 8.87. Found: C, 63.77; H, 7.06; N, 8.51.

5.5.5. $\quad N$-[4-[[4-[(3,4-Dihydro-6-hydroxy-2,2,7,8-tetramethyl-2H-1-benzopyran-5-yl)methyl|piperazin-1-yl]carbonyllphenyl|methanesulfonamide (5e). Yield: 54\%, white solid, mp $124-126{ }^{\circ} \mathrm{C}$. ${ }^{1} \mathrm{H}$ NMR $\delta: 7.34$ (d, 2H, $J=8.5 \mathrm{~Hz}), 7.22(\mathrm{~d}, 2 \mathrm{H}, J=8.5 \mathrm{~Hz}), 3.67-3.65(\mathrm{~m}$, $2 \mathrm{H}), 3.65(\mathrm{~s}, 2 \mathrm{H}), 3.47-3.45(\mathrm{~m}, 2 \mathrm{H}), 3.00(\mathrm{~s}, 3 \mathrm{H})$, 2.61-2.56 (m, 6H), $2.12(\mathrm{~s}, 3 \mathrm{H}), 2.09(\mathrm{~s}, 3 \mathrm{H}), 1.75(\mathrm{t}$, $2 \mathrm{H}, J=6.7 \mathrm{~Hz}), 1.28(\mathrm{~s}, 6 \mathrm{H}) .{ }^{13} \mathrm{C}$ NMR $\delta: 169.6$, $148.3,138.7,131.6,128.9,119.5,115.8,114.4,72.3$, 65.0, 56.1, 53.4, 52.3, 39.7, 33.0, 26.6, 20.8, 11.9, 11.7. Anal. Calcd for $\mathrm{C}_{26} \mathrm{H}_{35} \mathrm{~N}_{3} \mathrm{O}_{5} \mathrm{~S}$ : C, 62.25; H, 7.03; N, 8.38. Found: C, 62.27; H, 7.36; N, 8.66.

\section{6. (3,4-Dihydro-6-methoxy-2,2,7,8-tetramethyl-2H-1- benzopyran-5)-vinyl-methyl-ether (7)}

To a solution of $\mathrm{Ph}_{3} \mathrm{P}^{+} \mathrm{CH}_{2} \mathrm{OCH}_{3} \mathrm{Cl}^{-}(1.35 \mathrm{~g}, 3.9 \mathrm{mmol})$ in $8 \mathrm{~mL}$ anhyd THF was added, at $0^{\circ} \mathrm{C}, t$-BuOK $(0.295 \mathrm{~g}, 2.6 \mathrm{mmol})$, and the red mixture was stirred at $0{ }^{\circ} \mathrm{C}$ for $15 \mathrm{~min}$. A solution of aldehyde $6(0.320 \mathrm{~g}$, $1.29 \mathrm{mmol}$ ) in $8 \mathrm{~mL}$ THF was then added and the mixture was stirred at $0{ }^{\circ} \mathrm{C}$ for $15 \mathrm{~min}$ and at ambient temperature for $24 \mathrm{~h}$. Satd aqueous $\mathrm{NaHCO}_{3}$ was then added followed by extraction with diethyl ether. The organic layer was washed with satd aqueous $\mathrm{NaCl}$, dried and the solvent was evaporated. Purification by column chromatography (pet ether/AcOEt 8:2) afforded a mixture of cis/trans isomers. Yield: $0.330 \mathrm{~g}(93 \%)$, yellow oil. ${ }^{1} \mathrm{H} \quad \mathrm{NMR} \quad \delta$ (trans isomer): $7.09 \quad(\mathrm{~d}, 1 \mathrm{H}$, $\left.J=13.4 \mathrm{~Hz}, \quad-\mathrm{CH}=\mathrm{CH}-\mathrm{OCH}_{3}\right), \quad 5.71 \quad(\mathrm{~d}, \quad 1 \mathrm{H}, \quad J=$ $\left.13.4 \mathrm{~Hz},-\mathrm{CH}=\mathrm{CH}-\mathrm{OCH}_{3}\right), 3.72(\mathrm{~s}, 3 \mathrm{H},-\mathrm{CH}=\mathrm{CH}-$ $\left.\mathrm{OCH}_{3}\right), 3.61(\mathrm{~s}, 3 \mathrm{H}), 2.69(\mathrm{t}, 2 \mathrm{H}, J=6.7 \mathrm{~Hz}), 2.20(\mathrm{~s}$, $3 \mathrm{H}), 2.11(\mathrm{~s}, 3 \mathrm{H}), 1.77(\mathrm{t}, 2 \mathrm{H}, J=6.7 \mathrm{~Hz}), 1.31(\mathrm{~s}, 6 \mathrm{H})$.

\subsection{3,4-Dihydro-6-methoxy-2,2,7,8-tetramethyl-2H-1- benzopyran-5-acetaldehyde (8)}

To a solution of compound $7(0.330 \mathrm{~g}, 1.19 \mathrm{mmol})$ in $25 \mathrm{~mL} \mathrm{1,4-dioxane} \mathrm{and} 13 \mathrm{~mL} \mathrm{H}_{2} \mathrm{O}$, a catalytic amount of $p$-toluenesulfonic acid was added and the mixture is refluxed for $24 \mathrm{~h}$. After completion of the reaction, the mixture was extracted with diethyl ether and the organic layer was washed with satd aqueous $\mathrm{NaCl}$, dried, and the solvent was evaporated. Yield: $0.310 \mathrm{~g}(100 \%) .{ }^{1} \mathrm{H}$ NMR $\delta: 10.49(\mathrm{~s}, 1 \mathrm{H},-\mathrm{CHO}), 3.76(\mathrm{~s}, 3 \mathrm{H}), 3.59$ (s, $\left.2 \mathrm{H}, \mathrm{ArCH}_{2} \mathrm{CHO}-\right), 3.09(\mathrm{t}, 2 \mathrm{H}, J=6.5 \mathrm{~Hz}), 2.20(\mathrm{~s}$, $3 \mathrm{H}), 2.16(\mathrm{~s}, 3 \mathrm{H}), 1.73(\mathrm{t}, 2 \mathrm{H}, J=6.2 \mathrm{~Hz}), 1.29(\mathrm{~s}, 6 \mathrm{H})$.

\subsection{1-[2-(3,4-Dihydro-6-methoxy-2,2,7,8-tetramethyl-} 2H-1-benzopyran-5-yl)ethyl]-4-(4-nitrophenyl)piperazine (9)

To a solution of compound $8(0.094 \mathrm{~g}, 0.36 \mathrm{mmol})$ in $2 \mathrm{~mL} \mathrm{CH}{ }_{3} \mathrm{COOH}$ and $2 \mathrm{~mL} \mathrm{CH}_{3} \mathrm{CN}$, at $0{ }^{\circ} \mathrm{C}$, a solution of 1-(4-nitrophenyl)piperazine $(0.049 \mathrm{~g}, 0.24 \mathrm{mmol})$ in $2 \mathrm{~mL} \mathrm{CH} \mathrm{CH}_{3} \mathrm{COOH}$ was added and after stirring for
$10 \mathrm{~min}$ at $0{ }^{\circ} \mathrm{C}, \mathrm{NaBH}_{3} \mathrm{CN}(0.018 \mathrm{~g}, 0.29 \mathrm{mmol})$ was added and the mixture was stirred at ambient temperature for $24 \mathrm{~h}$. After completion of the reaction the mixture was poured into ice and $\mathrm{NaOH} 2 \mathrm{~N}$ was added until pH 6. The mixture was then extracted with AcOEt, the organic layer was washed with satd aqueous $\mathrm{NaCl}$, dried and the solvent evaporated. The residue was purified by column chromatography (AcOEt/pet. ether 8:2) Yield: $0.035 \mathrm{~g}(25 \%)$, orange viscous oil. ${ }^{1} \mathrm{H}$ NMR $\delta: 8.12(\mathrm{~d}$, $2 \mathrm{H}, J=8.5 \mathrm{~Hz}), 6.82(\mathrm{~d}, 2 \mathrm{H}, J=8.5 \mathrm{~Hz}), 3.67(\mathrm{~s}, 3 \mathrm{H})$, 3.49-3.47 (m, 4H), 2.82-2.79 (m, 2H), 2.71-2.52 (m, $8 \mathrm{H}), 2.17(\mathrm{~s}, 3 \mathrm{H}), 2.07(\mathrm{~s}, 3 \mathrm{H}), 1.76(\mathrm{t}, 2 \mathrm{H}$, $J=6.7 \mathrm{~Hz}), 1.27(\mathrm{~s}, 6 \mathrm{H})$. Anal. Calcd for $\mathrm{C}_{26} \mathrm{H}_{35} \mathrm{~N}_{3} \mathrm{O}_{4}$ : C, 68.85; H, 7.78; N, 9.26. Found: C, 69.11; H, 7.87; N, 9.64 .

\subsection{4-[4-[2-(3,4-Dihydro-6-methoxy-2,2,7,8-tetramethyl-} 2H-1-benzopyran-5-yl)ethyl|piperazin-1-yl|aniline (10)

This compound was prepared using method B. Yield: $0.028 \mathrm{~g}(98 \%)$, yellow viscous oil. ${ }^{1} \mathrm{H}$ NMR $\delta: 6.82$ (d, $2 \mathrm{H}, J=8.5 \mathrm{~Hz}), 6.61(\mathrm{~d}, 2 \mathrm{H}, J=8.5 \mathrm{~Hz}), 3.75-3.73$ $(\mathrm{m}, 2 \mathrm{H}), 3.67(\mathrm{~s}, 3 \mathrm{H}), 3.12-3.10(\mathrm{~m}, 2 \mathrm{H}), 2.82-2.80(\mathrm{~m}$, $2 \mathrm{H}), 2.73-2.51(\mathrm{~m}, 8 \mathrm{H}), 2.17(\mathrm{~s}, 3 \mathrm{H}), 2.07$ (s, 3H), 1.76 $(\mathrm{t}, 2 \mathrm{H}, J=6.7 \mathrm{~Hz}), 1.25(\mathrm{~s}, 6 \mathrm{H})$.

5.10. $N$-[4-[4-[2-(3,4-Dihydro-6-methoxy-2,2,7,8-tetramethyl-2H-1-benzopyran-5-yl)ethyl|piperazin-1-ylphenyl]methanesulfonamide (11)

This compound was prepared using method C. Yield: $0.029 \mathrm{~g}(41 \%)$ yellowish viscous oil. ${ }^{1} \mathrm{H}$ NMR $\delta: 7.15$ $(\mathrm{d}, 2 \mathrm{H}, J=8.5 \mathrm{~Hz}), 6.90(\mathrm{~d}, 2 \mathrm{H}, J=8.5 \mathrm{~Hz}), 3.67$ (s, $3 \mathrm{H}), 3.26-3.23(\mathrm{~m}, 4 \mathrm{H}), 2.93(\mathrm{~s}, 3 \mathrm{H}), 2.86-2.81(\mathrm{~m}$, 2H) $2.73-2.68(\mathrm{~m}, 6 \mathrm{H}), 2.56-2.52(\mathrm{~m}, 2 \mathrm{H}), 2.17(\mathrm{~s}$, $3 \mathrm{H}), 2.07(\mathrm{~s}, 3 \mathrm{H}), 1.77(\mathrm{t}, 2 \mathrm{H}, J=6.7 \mathrm{~Hz}), 1.29(\mathrm{~s}$, $6 \mathrm{H}) .{ }^{13} \mathrm{C}$ NMR $\delta: 149.9,148.2,128.2,124.6,124.1$, $117.0,116.7,72.8,61.2,58.4,53.0,48.9,38.9,32.9$, 26.9, 23.9, 20.4, 12.8, 11.9. Anal. Calcd for $\mathrm{C}_{27} \mathrm{H}_{39} \mathrm{~N}_{3} \mathrm{O}_{4}$ $\mathrm{S} \cdot \mathrm{H}_{2} \mathrm{O}: \mathrm{C}, 62.40 ; \mathrm{H}, 7.95 ; \mathrm{N}, 8.09$. Found: C, 61.98, H, 7.58; N, 7.73.

\subsection{1. $N$-[4-[4-[2-(3,4-Dihydro-6-hydroxy-2,2,7,8-tetra- methyl-2H-1-benzopyran-5-yl)ethyl|piperazin-1-yl]phen- yl]methanesulfonamide (12)}

This compound was prepared using method C. Yield: $0.010 \mathrm{~g}(40 \%)$, yellowish solid, $\mathrm{mp} 202-205^{\circ} \mathrm{C} .{ }^{1} \mathrm{H}$ NMR $\delta: 7.15(\mathrm{~d}, 2 \mathrm{H}, J=8.5 \mathrm{~Hz}), 6.91(\mathrm{~d}, 2 \mathrm{H}$, $J=8.5 \mathrm{~Hz}), 3.28-3.25(\mathrm{~m}, 4 \mathrm{H}), 2.93$ (s, 3H), 2.85-2.81 (m, 2H), 2.73-2.68 (m, 6H), 2.56-2.52 (m, 2H), $2.17(\mathrm{~s}$, $3 \mathrm{H}), 2.07(\mathrm{~s}, 3 \mathrm{H}), 1.77(\mathrm{t}, 2 \mathrm{H}, J=6.7 \mathrm{~Hz}), 1.28(\mathrm{~s}$, $6 \mathrm{H}) .{ }^{13} \mathrm{C}$ NMR $\delta: 149.2,148.2,128.0,124.6,123.8$, $117.0,116.4,72.5,61.0,58.4,48.6,38.9,32.9,26.9$, 23.7, 20.4, 12.1, 11.9. Anal. Calcd for $\mathrm{C}_{26} \mathrm{H}_{37} \mathrm{~N}_{3} \mathrm{O}_{4} \mathrm{~S}$ : C, 64.04; H, 7.65; N, 8.62. Found: C, 63.77; H, 7.73; $\mathrm{N}, 8.66$.

5.12. 3,4-Dihydro-6-methoxy-2,2,7,8-tetramethyl-5-(2nitroethenyl)-2H-1-benzopyran (13)

Aldehyde $6(0.200 \mathrm{~g}, 0.8 \mathrm{mmol})$ was added in a mixture of $3 \mathrm{~mL}$ anhyd $\mathrm{CH}_{3} \mathrm{NO}_{2}$ and cat amount of 
$\mathrm{CH}_{3} \mathrm{COONH}_{4}$. The mixture was stirred at $100^{\circ} \mathrm{C}$ for $2 \mathrm{~h}$. The solvent is then evaporated and $\mathrm{H}_{2} \mathrm{O}$ and a mixture of diethyl ether/ $\mathrm{CH}_{2} \mathrm{Cl}_{2}$ 9:1 were added. The organic layer was washed with $\mathrm{H}_{2} \mathrm{O}(2 \times 50 \mathrm{~mL}), \mathrm{HCl} 3 \mathrm{~N}(2 \times$ $25 \mathrm{~mL}$ ), and satd aqueous $\mathrm{NaCl}$, dried, and the solvent was evaporated. Yield: $0.223 \mathrm{~g}(96 \%)$, yellow solid, $\mathrm{mp}$ 101-103 ${ }^{\circ} \mathrm{C} .{ }^{1} \mathrm{H}$ NMR $\delta: 8.24(\mathrm{~d}, 1 \mathrm{H}, J=13.4 \mathrm{~Hz})$, $7.95(\mathrm{~d}, 1 \mathrm{H}, J=13.4 \mathrm{~Hz}), 3.62(\mathrm{~s}, 3 \mathrm{H}), 2.84(\mathrm{t}, 2 \mathrm{H}$, $J=6.7 \mathrm{~Hz}), 2.19(\mathrm{~s}, 3 \mathrm{H}), 2.13(\mathrm{~s}, 3 \mathrm{H}), 1.83(\mathrm{t}, 2 \mathrm{H}$, $J=6.7 \mathrm{~Hz}), 1.31(\mathrm{~s}, 6 \mathrm{H}) \mathrm{MS} m / z: 291\left(\mathrm{M}^{+}, 100 \%\right)$.

\subsection{2-(3,4-Dihydro-6-methoxy-2,2,7,8-tetramethyl-2H- 1-benzopyran-5-yl)ethylamine (14)}

To a slurry of $\mathrm{LiAlH}_{4}(0.087 \mathrm{~g}, 2.31 \mathrm{mmol})$ in $20 \mathrm{~mL}$ anhyd $\mathrm{THF}$ was added dropwise, at $0{ }^{\circ} \mathrm{C}$, a solution of compound $13(0.223 \mathrm{~g}, 0.77 \mathrm{mmol})$ in $20 \mathrm{~mL}$ anhyd $\mathrm{THF}$ and the mixture was refluxed for $2 \mathrm{~h}$. Some drops of $\mathrm{THF} / \mathrm{H}_{2} \mathrm{O}(1: 1)$ were then added to destroy the excess of $\mathrm{LiAlH}_{4}$. The mixture was diluted in AcOEt and $\mathrm{Na}_{2} \mathrm{SO}_{4}$ was added. Filtration through Celite and evaporation of the solvent afforded the desired amine. Yield: $0.200 \mathrm{~g}(98 \%)$, yellow oil. ${ }^{1} \mathrm{H}$ NMR $\delta: 3.62$ (s, 3H), 2.83$2.79(\mathrm{~m}, 2 \mathrm{H}), 2.75-2.72(\mathrm{~m}, 2 \mathrm{H}), 2.67(\mathrm{t}, 2 \mathrm{H}$, $J=6.7 \mathrm{~Hz}), 2.15(\mathrm{~s}, 3 \mathrm{H}), 2.06(\mathrm{~s}, 3 \mathrm{H}), 1.75(\mathrm{t}, 2 \mathrm{H}$, $J=6.7 \mathrm{~Hz}), 1.31(\mathrm{~s}, 6 \mathrm{H})$.

5.14. General procedure for the synthesis of secondary amines 15a,b (method E)

To a solution of 4-nitrophenethyl- or 2-(4-nitrophenoxy)ethylbromide $(0.5 \mathrm{mmol})$ in $5 \mathrm{~mL}$ anhyd $\mathrm{CH}_{3} \mathrm{CN}$ was added, at $0{ }^{\circ} \mathrm{C}, \mathrm{K}_{2} \mathrm{CO}_{3}(0.75 \mathrm{mmol})$, a solution of amine $14(0.5 \mathrm{mmol})$ in $5 \mathrm{~mL}$ anhyd $\mathrm{CH}_{3} \mathrm{CN}$, and a catalytic amount of TBAI, and the mixture was stirred at $50{ }^{\circ} \mathrm{C}$ for $24 \mathrm{~h}$. The solvent was then evaporated and the residue was extracted with $\mathrm{AcOEt}$ and $\mathrm{H}_{2} \mathrm{O}$. The organic layer was washed with satd aqueous $\mathrm{NaCl}$, dried, and evaporated to dryness. The residue was purified by column chromatography $\left(\mathrm{CH}_{2} \mathrm{Cl}_{2} / \mathrm{CH}_{3} \mathrm{OH}\right.$ 9.5:1.5).

5.14.1. $N$-[2-(4-Nitrophenyl)ethyl]-2-(3,4-dihydro-6-methoxy-2,2,7,8-tetramethyl-2H-1-benzopyran-5-yl)ethylamine (15a). Yield: $36 \%$, yellow viscous oil. ${ }^{1} \mathrm{H}$ NMR $\delta$ : $8.11(\mathrm{~d}, 2 \mathrm{H}, J=9.1 \mathrm{~Hz}), 7.31(\mathrm{~d}, 2 \mathrm{H}, J=9.1 \mathrm{~Hz}), 3.62$ $(\mathrm{s}, 3 \mathrm{H}), 2.93-2.90(\mathrm{~m}, 4 \mathrm{H}), 2.78-2.76(\mathrm{~m}, 4 \mathrm{H}), 2.66(\mathrm{t}$, $2 \mathrm{H}, J=6.7 \mathrm{~Hz}), 2.15(\mathrm{~s}, 3 \mathrm{H}), 2.07(\mathrm{~s}, 3 \mathrm{H}), 1.75(\mathrm{t}, 2 \mathrm{H}$, $J=6.7 \mathrm{~Hz}), 1.27(\mathrm{~s}, 6 \mathrm{H}) .{ }^{13} \mathrm{C}$ NMR $\delta: 149.7,148.2$, $148.0,146.5,129.5,128.1,127.4,124.2,123.6,117.0$, $72.8,60.9,50.3,49.6,36.1,32.8,26.9,26.8,20.4,12.8$, 11.9 .

5.14.2. $\quad N$-[2-(4-Nitrophenoxy)ethyl]-2-(3,4-dihydro-6methoxy-2,2,7,8-tetramethyl-2H-1-benzopyran-5-yl)ethylamine (15b). Yield: 42\%, yellowish viscous oil. ${ }^{1} \mathrm{H}$ NMR $\delta: 8.17(\mathrm{~d}, 2 \mathrm{H}, \quad J=9.1 \mathrm{~Hz}), 6.93 \quad(\mathrm{~d}, 2 \mathrm{H}$, $J=9.1 \mathrm{~Hz}), 4.16\left(\mathrm{t}, 2 \mathrm{H}, J=4.9 \mathrm{~Hz},-\mathrm{CH}_{2} \mathrm{CH}_{2} \mathrm{O}-\right) 3.65$ (s, 3H), $3.08\left(\mathrm{t}, 2 \mathrm{H}, J=4.9 \mathrm{~Hz},-\mathrm{CH}_{2} \mathrm{CH}_{2} \mathrm{O}-\right) 2.83$ $\left(\mathrm{m}, 4 \mathrm{H}, \quad \mathrm{ArCH}_{2} \mathrm{CH}_{2} \mathrm{NH}-\right), 2.69(\mathrm{t}, 2 \mathrm{H}, J=6.7 \mathrm{~Hz})$, $2.16(\mathrm{~s}, 3 \mathrm{H}), 2.07(\mathrm{~s}, 3 \mathrm{H}), 1.76(\mathrm{t}, 2 \mathrm{H}, J=6.7 \mathrm{~Hz}), 1.27$ $(\mathrm{s}, 6 \mathrm{H}) .{ }^{13} \mathrm{C} \mathrm{NMR} \delta$ : $163.9,149.7,148.2,141.5,128.1$, $127.3,125.9,124.2,117.0,114.5,72.8,68.3,65.0,49.9$, $48.2,32.8,27.1,26.9,20.4,12.8,11.9$.
5.15. General procedure for the synthesis of methylamines 16a,b (method F)

To $0.2 \mathrm{mmol}$ of the appropriate secondary amine were added at $0{ }^{\circ} \mathrm{C} \mathrm{HCOOH}(1 \mathrm{~mL})$ and $\mathrm{HCHO}$ $36 \%$ in water $(0.05 \mathrm{~mL})$ and the mixture was heated at $100{ }^{\circ} \mathrm{C}$ for $2 \mathrm{~h}$. $\mathrm{NaOH} 3 \mathrm{~N}$ was then added until $\mathrm{pH} 8$ and the mixture was extracted with AcOEt. The organic layer was washed with satd aqueous $\mathrm{NH}_{4} \mathrm{Cl}$, sat aqueous $\mathrm{NaCl}$, dried, and evaporated to dryness.

5.15.1. $N$-Methyl- $N$-[2-(4-nitrophenyl)ethyl]-2-(3,4-dihydro-6-methoxy-2,2,7,8-tetramethyl-2H-1-benzopyran-5yl)ethylamine (16a). Yield: 93\%, yellowish viscous oil. ${ }^{1} \mathrm{H}$ NMR $\delta: 8.13(\mathrm{~d}, 2 \mathrm{H}, J=8.5 \mathrm{~Hz}), 7.35(\mathrm{~d}, 2 \mathrm{H}$, $J=8.5 \mathrm{~Hz}), 3.66(\mathrm{~s}, 3 \mathrm{H}), 2.93-2.88(\mathrm{~m}, 2 \mathrm{H}) 2.77-$ $2.71(\mathrm{~m}, 4 \mathrm{H}), 2.67(\mathrm{t}, 2 \mathrm{H}, J=6.7 \mathrm{~Hz}), 2.57-2.52(\mathrm{~m}$, 2H), $2.43\left(\mathrm{~s}, 3 \mathrm{H},-\mathrm{N}\left(\mathrm{CH}_{3}\right)-\right) 2.17(\mathrm{~s}, 3 \mathrm{H}), 2.08(\mathrm{~s}$, $3 \mathrm{H}), \quad 1.76(\mathrm{t}, 2 \mathrm{H}, \quad J=6.7 \mathrm{~Hz}), 1.28(\mathrm{~s}, \quad 6 \mathrm{H}) .{ }^{13} \mathrm{C}$ NMR $\delta: 149.7,148.6,148.2,146.4,129.5,128.1$, $127.7,124.0,123.6,116.8,72.8,61.1,58.5,57.0$, $42.1,33.7,32.9,26.8,24.3,20.3,12.8,11.9$. MS m/z: $246(100 \%), 234,218$. Anal. Calcd for $\mathrm{C}_{25} \mathrm{H}_{34} \mathrm{~N}_{2} \mathrm{O}_{4}$ : C, 70.39; H, 8.03; N, 6.57. Found: C, 70.82; H, $8.35 ; \mathrm{N}, 6.73$.

5.15.2. $\quad N$-Methyl- $N$-[2-(4-nitrophenoxy)ethyl]-2-(3,4dihydro-6-methoxy-2,2,7,8-tetramethyl-2H-1-benzopyran5-yl)ethylamine (16b). Yield: 93\%, yellowish viscous oil. ${ }^{1} \mathrm{H}$ NMR $\delta: 8.17(\mathrm{~d}, 2 \mathrm{H}, J=9.1 \mathrm{~Hz}), 6.96(\mathrm{~d}, 2 \mathrm{H}$, $J=9.1 \mathrm{~Hz}), 4.17(\mathrm{t}, 2 \mathrm{H}, J=5.5 \mathrm{~Hz}) 3.65 \quad(\mathrm{~s}, 3 \mathrm{H})$, $2.93(\mathrm{t}, 2 \mathrm{H}, J=5.5 \mathrm{~Hz}) 2.82-2.77(\mathrm{~m}, 2 \mathrm{H}), 2.68(\mathrm{t}$, $2 \mathrm{H}, \quad J=6.7 \mathrm{~Hz}), 2.62-2.56(\mathrm{~m}, 2 \mathrm{H}), 2.48(\mathrm{~s}, 3 \mathrm{H})$, $2.17(\mathrm{~s}, 3 \mathrm{H}), 2.07(\mathrm{~s}, 3 \mathrm{H}), 1.76(\mathrm{t}, 2 \mathrm{H}, J=6.7 \mathrm{~Hz})$, $1.28(\mathrm{~s}, 6 \mathrm{H}) .{ }^{13} \mathrm{C}$ NMR $\delta: 163.9,149.7,148.2,141.5$, $132.0,128.1,127.5,125.8,124.0,116.8,114.5,72.8$, $67.1,65.0,61.1,57.8,55.7,50.8,42.8,32.9,26.8$, 24.3, 20.3, 12.8, 11.9. Anal. Calcd for $\mathrm{C}_{25} \mathrm{H}_{34} \mathrm{~N}_{2} \mathrm{O}_{5}$ : C, 67.85; H, 7.74; N, 6.33. Found: C, 67.92; H, 7.58; N, 6.32.

5.16. 4-[2-[N-Methyl-2-(3,4-dihydro-6-methoxy-2,2,7,8tetramethyl-2H-1-benzopyran-5-yl)ethylamine]ethyl]aniline (17a)

This compound was synthesized using method B. Yield: $85 \%$, yellowish viscous oil. ${ }^{1} \mathrm{H}$ NMR $\delta: 7.00(\mathrm{~d}, 2 \mathrm{H}$, $J=7.9 \mathrm{~Hz}), 6.62(\mathrm{~d}, 2 \mathrm{H}, J=7.9 \mathrm{~Hz}), 3.67(\mathrm{~s}, 3 \mathrm{H})$, 2.83-2.68 (m, 8H), $2.66(\mathrm{t}, 2 \mathrm{H}, J=6.7 \mathrm{~Hz}), 2.45(\mathrm{~s}$, $3 \mathrm{H}) 2.18(\mathrm{~s}, 3 \mathrm{H}), 2.08(\mathrm{~s}, 3 \mathrm{H}), 1.78(\mathrm{t}, 2 \mathrm{H}, J=6.7 \mathrm{~Hz})$, $1.29(\mathrm{~s}, 6 \mathrm{H})$.

5.17. 4-[2-[N-Methyl-2-(3,4-dihydro-6-methoxy-2,2,7,8tetramethyl- 2H-1-benzopyran-5-yl)ethylamine]ethoxy]aniline (17b)

This compound was synthesized using method B. Yield: $97 \%$, yellowish viscous oil. ${ }^{1} \mathrm{H}$ NMR $\delta: 6.75(\mathrm{~d}, 2 \mathrm{H}$, $J=8.5 \mathrm{~Hz}), 6.63(\mathrm{~d}, 2 \mathrm{H}, J=8.5 \mathrm{~Hz}), 4.06(\mathrm{t}, 2 \mathrm{H}$, $J=5.5 \mathrm{~Hz}), 3.66(\mathrm{~s}, 3 \mathrm{H}), 2.90-2.62(\mathrm{~m}, 8 \mathrm{H}), 2.49(\mathrm{~s}$, $\left.3 \mathrm{H},-\mathrm{NCH}_{3}\right), 2.17(\mathrm{~s}, 3 \mathrm{H}), 2.07(\mathrm{~s}, 3 \mathrm{H}), 1.76(\mathrm{t}, 2 \mathrm{H}$, $J=6.7 \mathrm{~Hz}), 1.28(\mathrm{~s}, 6 \mathrm{H})$. 
5.18. $N$-[4-[2-[|2-(3,4-Dihydro-6-methoxy-2,2,7,8-tetramethyl-2H-1-benzopyran-5-yl)ethyl|methylamine]ethyl]phenyl]methanesulfonamide (18a)

This compound was synthesized using method C. Yield: $70 \%$, yellowish viscous oil. ${ }^{1} \mathrm{H}$ NMR $\delta: 7.17-7.15(\mathrm{~m}$, $4 \mathrm{H}), 3.66(\mathrm{~s}, 3 \mathrm{H}), 2.97\left(\mathrm{~s}, 3 \mathrm{H},-\mathrm{NHSO}_{2} \mathrm{CH}_{3}\right), 2.81-$ $2.55(\mathrm{~m}, 10 \mathrm{H}), 2.45\left(\mathrm{~s}, 3 \mathrm{H},-\mathrm{NCH}_{3}\right), 2.17$ (s, 3H), 2.07 $(\mathrm{s}, 3 \mathrm{H}), 1.76(\mathrm{t}, 2 \mathrm{H}, J=6.7 \mathrm{~Hz}), 1.27(\mathrm{~s}, 6 \mathrm{H}) .{ }^{13} \mathrm{C}$ NMR $\delta$ : 149.7, 148.2, 137.9, 134.6, 129.9, 128.1, 127.6, $124.0,121.5,116.9,72.8,61.1,59.0,56.9,42.0,39.2$, 32.9, 26.9, 24.1, 20.3, 12.8, 11.9. Anal. Calcd for $\mathrm{C}_{26} \mathrm{H}_{38} \mathrm{~N}_{2} \mathrm{O}_{4} \mathrm{~S}$ : C, 65.79; H, 8.07; N, 5.90. Found: C, 65.92; H, 7.69; N, 6.04.

\subsection{9. $N$-[4-[2-[|2-(3,4-Dihydro-6-methoxy-2,2,7,8-tetra- methyl-2H-1-benzopyran-5-yl)ethyl|methylamine]ethoxy]- phenyl]methanesulfonamide (18b)}

This compound was synthesized using method C. Yield: $66 \%$, yellowish solid, mp $125-127^{\circ} \mathrm{C}$.

${ }^{1} \mathrm{H}$ NMR $\delta: 7.17(\mathrm{~d}, 2 \mathrm{H}, J=8.5 \mathrm{~Hz}), 6.86(\mathrm{~d}, 2 \mathrm{H}$, $J=8.5 \mathrm{~Hz}), 4.07(\mathrm{t}, 2 \mathrm{H}, J=5.5 \mathrm{~Hz}), 3.64(\mathrm{~s}, 3 \mathrm{H}), 2.91$ $(\mathrm{s}, 3 \mathrm{H}), 2.89-2.85(\mathrm{~m}, 2 \mathrm{H}), 2.81-2.76(\mathrm{~m}, 2 \mathrm{H}), 2.66(\mathrm{t}$, $2 \mathrm{H}, J=6.7 \mathrm{~Hz}), 2.60-2.56(\mathrm{~m}, 2 \mathrm{H}), 2.46(\mathrm{~s}, 3 \mathrm{H}) 2.15$ $(\mathrm{s}, 3 \mathrm{H}), 2.05(\mathrm{~s}, 3 \mathrm{H}), 1.77(\mathrm{t}, 2 \mathrm{H}, J=6.7 \mathrm{~Hz}), 1.26(\mathrm{~s}$, $6 \mathrm{H}) .{ }^{13} \mathrm{C}$ NMR $\delta: 157.3,149.7,148.2,129.1,128.1$, $127.5,124.7,124.0,116.9,115.5,72.8,66.4,61.1,57.8$, 55.9, 53.4, 42.7, 38.9, 32.9, 26.9, 24.1, 20.3, 12.8, 11.9. MS $m / z: 428,246(100 \%), 231$. Anal. Calcd for $\mathrm{C}_{26} \mathrm{H}_{38} \mathrm{~N}_{2} \mathrm{O}_{5} \mathrm{~S}$ : C, 63.65; H, 7.81; N, 5.71. Found: C, 63.88; H, 7.58; N, 5.35.

\subsection{0. $N$-[4-[2-[|2-(3,4-Dihydro-6-hydroxy-2,2,7,8-tetra- methyl-2H-1-benzopyran-5-yl)ethyl|methylamine]ethyl]- phenyl]methanesulfonamide (19a)}

This compound was synthesized using method D. Yield: $53 \%$, yellowish viscous oil. ${ }^{1} \mathrm{H}$ NMR $\delta: 7.12-7.10(\mathrm{~m}$, $4 \mathrm{H}), 2.96(\mathrm{~s}, 3 \mathrm{H}), 2.76-2.67(\mathrm{~m}, 8 \mathrm{H}), 2.61(\mathrm{t}, 2 \mathrm{H}$, $J=6.7 \mathrm{~Hz}), 2.45(\mathrm{~s}, 3 \mathrm{H}) 2.16(\mathrm{~s}, 3 \mathrm{H}), 2.10(\mathrm{~s}, 3 \mathrm{H}), 1.77$ $(\mathrm{t}, 2 \mathrm{H}, J=6.7 \mathrm{~Hz}), 1.27(\mathrm{~s}, 6 \mathrm{H}) .{ }^{13} \mathrm{C} \mathrm{NMR} \delta: 148.1$, $145.0,137.8,135.0,129.9,128.0,127.5,123.9,121.4$, $115.7,72.2,60.1,53.4,42.2,39.2,33.2,26.7,24.0,21.1$, 12.5, 12.0. Anal. Calcd for $\mathrm{C}_{25} \mathrm{H}_{36} \mathrm{~N}_{2} \mathrm{O}_{4} \mathrm{~S}$ : C, 65.19; $\mathrm{H}$, 7.88; N, 6.08. Found: C, 64.82; H, 7.73; N, 6.41.

\subsection{1. $N$-[4-[2-[|2-(3,4-Dihydro-6-hydroxy-2,2,7,8-tetra- methyl-2H-1-benzopyran-5-yl)ethyl|methylamine]ethoxyl- phenyl]methanesulfonamide (19b)}

This compound was synthesized using method D. Yield: $37 \%$, yellowish viscous oil. ${ }^{1} \mathrm{H}$ NMR $\delta: 7.15(\mathrm{~d}, 2 \mathrm{H}$, $J=8.5 \mathrm{~Hz}), 6.79(\mathrm{~d}, 2 \mathrm{H}, J=8.5 \mathrm{~Hz}), 4.08(\mathrm{t}, 2 \mathrm{H}$, $J=5.5 \mathrm{~Hz}), 2.93(\mathrm{~s}, 3 \mathrm{H}), 2.90-2.87(\mathrm{~m}, 2 \mathrm{H}), 2.82-2.78$ $(\mathrm{m}, 4 \mathrm{H}), 2.59(\mathrm{t}, 2 \mathrm{H}, J=6.7 \mathrm{~Hz}), 2.54(\mathrm{~s}, 3 \mathrm{H}) 2.14(\mathrm{~s}$, $3 \mathrm{H}), 2.05(\mathrm{~s}, 3 \mathrm{H}), 1.74(\mathrm{t}, 2 \mathrm{H}, J=6.7 \mathrm{~Hz}), 1.25(\mathrm{~s}$, $6 \mathrm{H}) .{ }^{13} \mathrm{C}$ NMR $\delta: 151.2,149.7,148.2,129.5,128.0$, $127.3,125.1,124.0,116.7,115.3,72.8,66.4,61.0,57.8$, 55.5, 42.7, 38.9, 32.9, 26.9, 24.3, 20.3, 12.2, 11.9. Anal. Calcd for $\mathrm{C}_{25} \mathrm{H}_{36} \mathrm{~N}_{3} \mathrm{O}_{5} \mathrm{~S}: \mathrm{C}, 63.00 ; \mathrm{H}, 7.61 ; \mathrm{N}, 5.88$. Found: C, 62.79; H, 8.01; N, 5.53.
5.22. 3,4-Dihydro-6-methoxy-5-[(6-nitro-2,3-dihydro-1 $H$ indol-1-yl)methyll-2,2,7,8-tetramethyl-2 $H$-1-benzopyran (20)

The synthesis of this compound was carried out following method A. Yield: 93\%, orange solid, mp $145-147^{\circ} \mathrm{C}$. ${ }^{1} \mathrm{H}$ NMR $\delta: 7.52(\mathrm{~d}, 1 \mathrm{H}, J=7.93 \mathrm{~Hz}), 7.34(\mathrm{~s}, 1 \mathrm{H}), 7.08$ $(\mathrm{d}, 1 \mathrm{H}, J=7.9 \mathrm{~Hz}), 4.26(\mathrm{~s}, 2 \mathrm{H}), 3.64(\mathrm{~s}, 3 \mathrm{H}), 3.28(\mathrm{t}$, $2 \mathrm{H}, J=8.6 \mathrm{~Hz}), 2.91(\mathrm{t}, 2 \mathrm{H}, J=8.6 \mathrm{~Hz}), 2.74(\mathrm{t}, 2 \mathrm{H}$, $J=6.7 \mathrm{~Hz}), 2.20(\mathrm{~s}, 3 \mathrm{H}), 2.12(\mathrm{~s}, 3 \mathrm{H}), 1.75(\mathrm{t}, 2 \mathrm{H}$, $J=6.7 \mathrm{~Hz}), 1.29(\mathrm{~s}, 6 \mathrm{H}) .{ }^{13} \mathrm{C}$ NMR $\delta: 156.2,153.4$, $150.4,148.6,148.2,138.2,128.2,126.1,124.0,118.2$, $113.3,100.2,73.1,61.5,52.4,43.6,38.5,32.7,28.2$, 26.9, 20.0, 12.9, 12.1. MS m/z: $396\left(\mathrm{M}^{+}\right), 233,217$. Anal. Calcd for $\mathrm{C}_{23} \mathrm{H}_{28} \mathrm{~N}_{2} \mathrm{O}_{4}$ : C, 69.68; H, 7.12; N, 7.07. Found: C, 69.96; H, 6.92; N, 7.32.

5.23. 3,4-Dihydro-6-methoxy-5-[(6-nitro-2,3-dihydro-1 $H$ indol-1-yl)ethyl|-2,2,7,8-tetramethyl-2H-1-benzopyran (21)

Aldehyde 8 was treated with 6-nitroindoline as described for analogue 9. Yield: $35 \%$, yellowish viscous oil ${ }^{1} \mathrm{H}$ NMR $\delta: 7.50(\mathrm{~d}, 1 \mathrm{H}, J=7.9 \mathrm{~Hz}), 7.26(\mathrm{~s}, 1 \mathrm{H}), 7.07(\mathrm{~d}$, $1 \mathrm{H}, J=7.9 \mathrm{~Hz}), 3.72(\mathrm{~s}, 3 \mathrm{H}), 3.62(\mathrm{t}, 2 \mathrm{H}, J=8.6 \mathrm{~Hz})$, $3.28(\mathrm{t}, 2 \mathrm{H}, J=7.9 \mathrm{~Hz}), 3.05(\mathrm{t}, 2 \mathrm{H}, J=8.6 \mathrm{~Hz}), 2.82$ $(\mathrm{t}, 2 \mathrm{H}, J=7.9 \mathrm{~Hz}), 2.72(\mathrm{t}, 2 \mathrm{H}, J=6.7 \mathrm{~Hz}), 2.21(\mathrm{~s}$, $3 \mathrm{H}), 2.09(\mathrm{~s}, 3 \mathrm{H}), 1.79(\mathrm{t}, 2 \mathrm{H}, J=6.7 \mathrm{~Hz}), 1.29(\mathrm{~s}$, $6 \mathrm{H}) .{ }^{13} \mathrm{C}$ NMR $\delta$ : $155.8,152.9,150.7,148.0,136.3$, $128.4,125.1,122.3,118.5,112.3,101.2,72.9,61.0$, $53.5,44.6,37.2,33.5,31.8,28.4,25.9,20.3,12.6,11.9$.

5.24. $N-\{1-[(3,4-D i h y d r o-6-h y d r o x y-2,2,7,8-t e t r a m e t h y l-$ 2H-1-benzopyran-5-yl)methyl]-2,3-dihydro-1 $H$-indol-6yl\}methanesulfonamide (22)

The synthesis of this compound was carried out using method D. Yield: 33\%, yellowish solid, mp 149$152{ }^{\circ} \mathrm{C} .{ }^{1} \mathrm{H}$ NMR $\delta: 7.09(\mathrm{~d}, 1 \mathrm{H}, J=7.9 \mathrm{~Hz}), 6.70(\mathrm{~d}$, $1 \mathrm{H}, J=6.7 \mathrm{~Hz}), 6.59(\mathrm{~s}, 1 \mathrm{H}), 4.25(\mathrm{~s}, 2 \mathrm{H}), 3.30(\mathrm{t}, 2 \mathrm{H}$, $J=7.9 \mathrm{~Hz}), 2.94(\mathrm{~m}, 5 \mathrm{H}), 2.66(\mathrm{t}, 2 \mathrm{H}, J=6.7 \mathrm{~Hz})$, $2.14(\mathrm{~s}, 3 \mathrm{H}), 2.09$ (s, 3H), $1.78(\mathrm{t}, 2 \mathrm{H}, J=6.7 \mathrm{~Hz}), 1.29$ $(\mathrm{s}, 6 \mathrm{H}) .{ }^{13} \mathrm{C}$ NMR $\delta$ : $153.3,151.0,149.7,144.5,135.6$, $124.2,121.5,120.0,118.3,117.6,106.2,100.3,72.9$, 61.5, 53.1, 40.1, 38.5, 31.4, 27.7, 26.9, 20.5, 12.1, 11.8 Anal. Calcd for $\mathrm{C}_{23} \mathrm{H}_{30} \mathrm{~N}_{2} \mathrm{O}_{4} \mathrm{~S}: \mathrm{C}, 64.16 ; \mathrm{H}, 7.02 ; \mathrm{N}$, 6.51. Found: C, 63.88; H, 6.97; N, 6.35.

5.25. $N$-\{1-[(3,4-Dihydro-6-hydroxy-2,2,7,8-tetramethyl2H-1-benzopyran-5-yl)ethyl|-2,3-dihydro-1 $H$-indol-6yl\}methanesulfonamide (23)

This compound was synthesized using method D. Yield: $29 \%$, yellowish viscous oil. ${ }^{1} \mathrm{H}$ NMR $\delta: 7.01(\mathrm{~d}, 1 \mathrm{H}$, $J=7.9 \mathrm{~Hz}), 6.52(\mathrm{~d}, 1 \mathrm{H}, J=6.7 \mathrm{~Hz}), 6.46(\mathrm{~s}, 1 \mathrm{H}), 3.46$ $(\mathrm{t}, 2 \mathrm{H}, J=7.9 \mathrm{~Hz}), 3.27(\mathrm{t}, 2 \mathrm{H}, J=6.7 \mathrm{~Hz}), 2.93(\mathrm{~m}$, $7 \mathrm{H}), 2.70(\mathrm{t}, 2 \mathrm{H}, J=6.7 \mathrm{~Hz}), 2.12(\mathrm{~s}, 3 \mathrm{H}), 2.09(\mathrm{~s}$, $3 \mathrm{H}), 1.81(\mathrm{t}, 2 \mathrm{H}, J=6.7 \mathrm{~Hz}), 1.29(\mathrm{~s}, 6 \mathrm{H}) .{ }^{13} \mathrm{C} \mathrm{NMR}$ $\delta: 155.3,151.0,148.2,143.5,133.1,124.0,121.1,120.3$, $118.8,116.3,107.5,101.3,72.5,61.3,52.1,40.3,38.5$, $31.4,28.5,27.1,26.3,20.5,12.4,11.9$ Anal. Calcd for $\mathrm{C}_{24} \mathrm{H}_{32} \mathrm{~N}_{2} \mathrm{O}_{4} \mathrm{~S}$ : C, 64.84; H, 7.25; N, 6.30. Found: C, 64.82; H, 7.41; N, 6.73. 


\subsection{Biology}

5.26.1. Evaluation of the activity of the new compounds against reperfusion arrhythmias. Male Sprague-Dawley rats weighing about $300-350 \mathrm{~g}$ were housed under controlled light (12L:12D) and temperature with free access to food and water in compliance with the prescriptions for the care and use of laboratory animals. Rats were anesthetized with pentobarbital (30-40 mg per animal). After intravenous administration of heparin, the chests were opened the hearts were rapidly excised and mounted on a non-recirculating Langendorff perfusion apparatus. Retrograde perfusion was established at a pressure of $90 \mathrm{~cm} \mathrm{H}_{2} \mathrm{O}$ with an oxygenated normothermic Krebs-Hensleit bicarbonate (KHB) buffer $\left(25 \mathrm{mmol} \mathrm{L}^{-1}\right.$ $\mathrm{NaHCO}_{3}, 118 \mathrm{mmol} \mathrm{L}^{-1} \mathrm{NaCl}, 2.5 \mathrm{mmol} \mathrm{L}{ }^{-1} \mathrm{CaCl}_{2}$, $4.7 \mathrm{mmol} \mathrm{L}^{-1} \quad \mathrm{KCl}, \quad 1.4 \mathrm{mmol} \mathrm{L}^{-1} \quad \mathrm{MgSO}_{4}$, and $1.2 \mathrm{mmol} \mathrm{L}^{-1} \mathrm{KH}_{2} \mathrm{PO}_{4}$, pH 7.2, at $25^{\circ} \mathrm{C}$ ) supplemented with $11 \mathrm{mmol} \mathrm{L}^{-1}$ glucose and equilibrated with $95 \%$ $\mathrm{O}_{2} / 5 \% \mathrm{CO}_{2}$. The temperature of the hearts and perfusates was maintained at $37^{\circ} \mathrm{C}$ by the use of a waterjacketed apparatus. All hearts were equilibrated for $20 \mathrm{~min}$ under these conditions. At the end of the equilibration period, hearts were made ischemic for $10 \mathrm{~min}$ by perfusing them with the ischemic KHB (KHB with Tris-HCl $10 \mathrm{mM}, \mathrm{pH} 6.4$, instead of glucose and equilibrated with $\mathrm{N}_{2}$ before use) followed by $15 \mathrm{~min}$ of reperfusion. The new compounds were present during ischemia and reperfusion at a final concentration of $10 \mu \mathrm{M}$.

5.26.2. Evaluation of antiarrhythmic activity. Electrocardiograms were recorded during equilibration, ischemia, and reperfusion. Arrhythmias were scored according to the Lambeth Convention Guidelines. ${ }^{22}$ Arrhythmia scores (AS) were calculated for the first $10 \mathrm{~min}$ of reperfusion as the percentage of premature beats.

5.26.3. Evaluation of antioxidant activity. At the end of the perfusions, hearts were 'freeze-clamped' between aluminum tongs, cooled in liquid $\mathrm{N}_{2}$ and after the removal of the atria, the ventricles were pulverized under liquid $\mathrm{N}_{2}$ and powders were stored at $-80{ }^{\circ} \mathrm{C}$. A portion of the tissue powder was analyzed for malondialdehyde (MDA) content by using the thiobarbituric acid assay. ${ }^{23}$ To prevent auto-oxidation of the samples, homogenization was carried out at $4{ }^{\circ} \mathrm{C}$ in nitrogen equilibrated solution in the presence of $0.04 \%$ butylated hydroxytoluene, $1.6 \%$ ethanol. The values were expressed as nanomoles of TBA reactive substances (MDA equivalent) per gram of tissue. 1,1,3,3-Tetraethoxypropane $(0,0.5,1.0,2.0,4.0,8.0$ and $16.0 \mathrm{nmol})$ served as external standard. Results are expressed as mean$\mathrm{s} \pm$ SEM. Differences between groups were assessed by Student's unpaired and ANOVA $t$-tests and considered significant when $p<0,05$.

5.26.4. Investigation of the new analogues by a conventional microelectrode method. New Zeeland rabbits (1.5$2 \mathrm{~kg}$ ) were anesthetized by pentabarbital (iv $50 \mathrm{mg} / \mathrm{kg}$ ). After chest was opened, hearts were immediately rinsed in oxygenated Tyrode's solution containing (in $\mathrm{mM}$ ): $\mathrm{NaCl}, 115 ; \mathrm{KCl} 5 ; \mathrm{CaCl}_{2}$ 1.2; $\mathrm{MgCl}_{2} 1 ; \mathrm{NaHCO}_{3} 21.4$; and glucose 11. The $\mathrm{pH}$ of this solution is 7.35-7.45 when gassed with $95 \% \mathrm{O}_{2}$ and $5 \% \mathrm{CO}_{2}$ at $37^{\circ} \mathrm{C}$. The tip of the papillary muscles from the right ventricle was prepared and individually mounted in a tissue chamber (volume $\approx 50 \mathrm{~mL}$ ).

The preparations were continuously stimulated (by HSE [Hugo Sachs Electronik] Stimulator type 215/II, MarchHugstetten, Germany) at a basic cycle length of $1000 \mathrm{~ms}$ using $2 \mathrm{~ms}$ long rectangular constant voltage pulses isolated from ground and delivered across bipolar platinum electrodes in contact with the preparation. At least $1 \mathrm{~h}$ was allowed for each preparation to equilibrate while they were continuously superfused with Tyrode's solution. Temperature of the superfusate was kept constant at $37^{\circ} \mathrm{C}$.

Transmembrane potentials were recorded using conventional microelectrode techniques. Microelectrodes filled with $3 \mathrm{M} \mathrm{KCl}$ and having tip resistances of 5-20 M $\Omega$ were connected to the input of a high impedance electrometer (Experimetria Microelectrode Amplifier Type), which was referenced to the ground. The voltage outputs from the amplifier was displayed on a dual beam memory oscilloscope (Tektonix $2230100 \mathrm{MHz}$ Digital Storage Oscilloscope, Beaverton, OR, USA).

The maximal diastolic potential (MDP) ('resting potential,' RP), action potential amplitude (APA), the first derivative of transmembrane potentials $\left(V_{\max }\right)$ and action potential duration measured at $50 \%$ and $90 \%$ repolarization $\left(\mathrm{APD}_{50-90}\right)$, were obtained using a software developed in the Department of Pharmacology, Univ, of Szeged (HSE-APES). After the control measurements, the compounds were added to the tissue bath at $5 \mu \mathrm{M}$ concentration and the measurements were prepared after 30-40 min incubation time. When the impalement was lost during measurement, readjustment was attempted. If the difference between the original and the readjusted action potential parameters did not exceed $15 \%$, the experiment was continued, otherwise it was terminated.

\section{Acknowledgments}

This work was supported by the Greek General Secretariat for Research and Technology, grant Bilateral Collaboration of Greece-Hungary 736, by the National Institute of Health, DA3801 and by the Hungarian National Research Foundation, OTKA T-048698.

\section{References and notes}

1. Pugsley, M. K. Drug Dev. Res. 2002, 55, 3-16.

2. (a) Ravens, U.; Wettwer, E.; Hála, O. Cell Calcium 2004, 35, 575-582; (b) Tamargo, J.; Caballero, R.; Gómez, R.; Valenzuela, C.; Delpón, G. Cardiovasc. Res. 2004, 62, 933.

3. Carmeliet, E. Physiol. Rev. 1999, 79, 917-987.

4. (a) Lefer, D. J.; Granger, N. Am. J. Med. 2000, 109, 315323; (b) Vergely, C.; Maupoil, V.; Clermont, G.; Bril, A.; Rochette, L. Arch. Biochem. Biophys. 2003, 420, 209-216. 
5. (a) DiDomenico, R. J.; Massad, M. G. Ann. Thorac. Surg. 2005, 79, 728-740; (b) Knotzer, H.; Dunser, M. W.; Mayr, A. J.; Hasibeder, W. R. Curr. Opin. Crit. Care 2004, 10, 330-335.

6. Elming, H.; Brendorp, B.; Pehrson, S.; Pedersen, O. D.; Kober, L.; Torp-Petersen, C. Expert Opin. Drug Saf. 2004, 3, 559-577.

7. Nattel, S.; Singh, B. N. Am. J. Cardiol. 1999, 84, 11R19R.

8. Koster, R. W. N. Eng. J. Med. 1985, 313, 1105-1110.

9. Li, G.-R.; Ferrier, G. R. J. Pharmacol. Exp. Ther. 1991, 257, 997-1004.

10. Nair, L. A.; Grant, A. O. Cardiovasc. Drugs Ther. 1997, 11, 149-167.

11. (a) Rochetaing, A.; Barbe, C.; Kreher, P. J. Cardiovasc. Pharmacol. 2001, 38, 500-511; (b) Dogrell, S. A.; Hancox, J. C. Expert Opin. Investig. Drugs 2004, 13, 415-426; (c) Tse, H. F.; Lau, C. P. J. Am. Coll. Cardiol. 2002, 40, 21502155 .

12. (a) Brendorp, B.; Pedersen, O.; Torp-Pedersen, C.; Sahebzadah, N.; Kober, L. Drug Saf. 2002, 25, 847865; (b) Purerfellner, H. Curr. Med. Chem. Cardiovasc. Hematol. Agents 2004, 2, 79-91; (c) Kathofer, S.; Thomas, D.; Karle, C. A. Cardiovasc. Drug Rev. 2005, 23, 217-230.
13. Carlson, M. Expert Rev. Cardiovasc. Ther. 2005, 3, 387391.

14. Sager, P. T. Curr. Opin. Cardiol. 2000, 15, 41-53.

15. Vos, M. A. J. Cardiovasc. Electrophysiol. 2001, 12, 1034 1036.

16. MacKenzie, I. Drug Dev. Res. 2002, 55, 73-78.

17. Koufaki, M.; Calogeropoulou, T.; Rekka, E.; Chryselis, M.; Papazafiri, P.; Gaitanaki, C.; Makriyannis, A. Bioorg. Med. Chem. 2003, 11, 5209-5219.

18. Koufaki, M.; Detsi, A.; Theodorou, E.; Kiziridi, C.; Calogeropoulou, T.; Vassilopoulos, A.; Kourounakis, A.; Rekka, E.; Kourounakis, P.; Gaitanaki, C.; Papazafiri, P. Bioorg. Med. Chem. 2004, 12, 4835-4841.

19. Novák, J.; Salemink, C. A. Tetrahedron Lett. 1981, 22, 1063-1064.

20. Monte, A. P.; Waldman, S. R.; Marona-Lewicka, D.; Wainscott, D. B.; Nelson, D. L.; Sanders-Bush, E.; Nichols, D. E. J. Med. Chem. 1997, 40, 2997-3008.

21. Liu, H.; JI, M.; Luo, X.; Shen, J.; Huang, X.; Hua, W.; Jiang, H.; Chen, K. J. Med. Chem. 2002, 45, 29532969.

22. Walker, M.; Curtis, M.; Hearse, D. Cardiol. Res. 1988, 22, 447-455.

23. Draper, H. H.; Hardley, M. Methods Enzymol. 1990, 186, 283-293. 